\title{
A distance-limited continuous location-allocation problem for spatial planning of decentralized systems
}

\author{
Kagan Gokbayrak*, Ayse Selin Kocaman \\ Department of Industrial Engineering, Bilkent University, 06800 Bilkent, Ankara, Turkey
}

\section{A R T I C L E I N F O}

\section{Article history:}

Received 25 July 2016

Revised 14 June 2017

Accepted 17 June 2017

Available online 19 June 2017

\section{Keywords:}

Continuous location-allocation

Planar set covering

Multi-source Weber problem

Decentralized systems

\begin{abstract}
A B S T R A C T
We introduce a new continuous location-allocation problem where the facilities have both a fixed opening cost and a coverage distance limitation. The problem has wide applications especially in the spatial planning of water and/or energy access networks where the coverage distance might be associated with the physical loss constraints. We formulate a mixed integer quadratically constrained problem (MIQCP) under the Euclidean distance setting and present a three-stage heuristic algorithm for its solution: In the first stage, we solve a planar set covering problem (PSCP) under the distance limitation. In the second stage, we solve a discrete version of the proposed problem where the set of candidate locations for the facilities is formed by the union of the set of demand points and the set of locations in the PSCP solution. Finally, in the third stage, we apply a modified Weiszfeld's algorithm with projections that we propose to incorporate the coverage distance component of our problem for fine-tuning the discrete space solutions in the continuous space. We perform numerical experiments on three example data sets from the literature to demonstrate the performance of the suggested heuristic method.
\end{abstract}

(C) 2017 Elsevier Ltd. All rights reserved.

\section{Introduction}

Source location and allocation problems are the essential components of strategic planning for sustainable development. Many problems have been studied to help decision making in this area. Some of these studies included a list of predetermined candidate locations to locate source facilities, thus solved site-selecting location problems in a discrete space. Greenfield development problems, however, involves undeveloped sites that have no existing infrastructure and the facilities can be located at any point on a continuous space. This type of facility location problems are known as the site-generating problems (Love et al., 1988).

Motivated by the popularity of the decentralized systems in the energy and the water access networks, in this paper, we study a site-generating location-allocation problem for greenfield infrastructure planning. Our aim is to determine the number and the locations of the source facilities, which can be, for example, a solar or a wind power generation system or a water pump serving demand points as a stand-alone system. Assuming that the energy or the water resource availability is even over the field, the locationallocation decisions are made based on the spatial locations of the demand points. Our objective is to minimize the sum of the facility opening costs, which are independent of the locations of the

\footnotetext{
* Corresponding author.

E-mail addresses: kgokbayr@bilkent.edu.tr (K. Gokbayrak), selin.kocaman@bilkent.edu.tr (A.S. Kocaman).

facilities, and the connection costs to serve demand points such as cable or pipe installation costs that are linearly increasing in the distances to the serving facilities. All facilities are assumed to be uncapacitated; however, they can only serve demand points within a specified distance. This coverage distance limitation of the facilities can be associated with the constraints on the voltage drop in the energy systems (due to the resistance on cables) as in Kocaman et al. (2012) or the pressure loss in the water systems (due to the friction in the pipes) as in Douglas et al. (1979) that are both linearly increasing with distance.

We present and study a continuous location-allocation problem with a fixed facility opening cost and a limit on the coverage distance of the facilities. This problem is related to three well-known problems in the literature: the planar set covering problem (PSCP), the uncapacitated multi-source Weber problem (MWP), and the simple plant location problem (SPLP). In the special case, where there is no connection costs between the demand points and their serving facilities, our problem reduces to the PSCP. The original set covering problem (SCP) considers a finite collection of sets and their costs, and determines the lowest cost sub-collection whose union equals the union of the collection. This problem is known to be an NP-hard problem (Garey and Johnson, 1979). Several exact (Balas and Carrera, 1996; Beasley, 1987; Beasley and K.Jörnsten, 1992; Fisher and Kedia, 1990) and heuristic (Beasley, 1990; Beasley and Chu, 1996; Caprara et al., 1999; Haddadi, 1997; Lorena and Lopes, 1994) methods are proposed to solve the SCP that have 
applications in fields such as crew scheduling (e.g., Caprara et al., 1999) and locating emergency facilities (e.g., Rajagopalan et al., 2008; Toregas et al., 1971). The algorithms for the SCP are compared in the survey paper (Caprara et al., 2000) by Caprara et al. After the turn of the century, the work on the SCP concentrate on heuristic algorithms based on greedy randomized search (Bautista and Pereira, 2007; Haouari and Chaouachi, 2002; Lan et al., 2007), local search (Yagiura et al., 2006), genetic algorithm (Solar et al., 2002) and ant colony optimization (Ren et al., 2010). The PSCP problem considers a finite number of demand points in the Euclidean space and determines the minimum number of facilities and their locations in the plane such that each demand point is within a certain distance to at least one of these facilities. To solve the PSCP exactly, Church (1984) defined the circle intersection points set (CIPS) as the locations of all demand points and the intersection points of all circles centered at the demand points with a radius of a predetermined coverage distance. Then, for each point in the CIPS, a set is formed of all demand points that are within the coverage distance from the point. Considering the collection of all these sets, the original version of the SCP is solved. It is possible to show that there exists at least one optimal solution to the PSCP in which all facilities are located in the CIPS (Eiselt and Sandblom, 2013).

The MWP is a site-generating location-allocation problem, which is also known as the continuous p-median problem. It locates $p$ facilities in the Euclidean plane to serve a finite set of demand points, each having an associated weight. In this problem, each demand point is served by the closest facility and the objective is to minimize the weighted sum of the distances to the closest facilities. The MWP is known to be an NP-hard problem (Megiddo and Supowit, 1984); therefore, several heuristic solution methods are proposed in the literature. Cooper's iterative locationallocation algorithm (Cooper, 1963; 1964) is a well-known algorithm developed for this problem. Starting at an arbitrary solution that divides the set of demand points into $p$ almost-equal-sized subsets, the algorithm alternates between location and allocation steps until a local optimal solution is found. In the allocation step, for fixed locations of the facilities, algorithm simply assigns each demand point to its nearest facility (breaking ties arbitrarily), and once the allocations are fixed, in the location step, the problem reduces to $p$ independent single facility location problems that can be solved by the modified Weiszfeld's method in Vardi and Zhang (2001). As the final solution depends on the initial solution, a random multi-start version of this algorithm can be applied as in Drezner et al. (2016). Another line of work is based on the idea of starting at a good initial solution. Based on the observation that the optimal solution of the continuous problem often has several facilities co-located with the demand points, in Hansen et al. (1998) proposed the p-median heuristic. This heuristic first solves the p-median problem, which chooses $p$ facility locations from the set of demand points to minimize the weighted sum of distances. Then, $p$ independent single facility location problems are solved as in the location step of the Cooper's algorithm. Recently, Brimberg and Drezner (2013) proposed to overlay the area containing the demand points with a grid. Then, a p-median problem is solved over the nodes of the grid to obtain high-quality starting points for the Cooper's algorithm. Since there is a significant correlation between the qualities of the initial and the final solutions, starting at the p-median solution improves the algorithm results. Brimberg et al. (2014) proposed an alternating solution procedure where a local search is conducted in the continuous space to obtain a local optimum. The locations from the continuous problem solution is then augmented in the discrete space problem, which is solved again to obtain new initial points for the continuous space problem. This process continues until no further improvement is observed. Finally, Drezner et al. (2015) developed a distribution-based variable neighborhood search and a genetic algorithm, and a hybrid algorithm that combines these two approaches. The hybrid approach outperformed both approaches. For other heuristic, metaheuristic and exact approaches for the MWP, readers can refer to a comprehensive review by Brimberg et al. (2008).

The SPLP is a problem in a discrete space, where there are fixed facility opening costs and a finite set of possible locations for the facilities. It aims to minimize the sum of the facility opening costs and the weighted connection costs. The adjective "simple" in its name is to state that the facilities are uncapacitated. This problem is widely studied in the literature. Krarup and Pruzan (1983) provided a highly cited survey on this problem. It is stated in that paper that the SPLP is also an NP-hard problem. The version of SPLP with distance constraints also appeared in the literature. Berman and Yang (1991) introduced the problem and proposed an iterative algorithm starting from the solution of the uncapacitated facility location problem. Krysta and Solis-Oba. (2001) and Weng (2013) presented integer programming (IP) formulations for the unweighted problem and proposed approximation algorithms. The work on the continuous space version of the SPLP, however, is very limited. Brimberg et al. (2004) introduced the fixed cost for facilities that is independent of the location. The problem that we consider in this paper reduces to the problem considered in Brimberg et al. (2004) if the coverage distance limitation is removed. They proposed a multi-stage heuristic approach for the problem without the coverage constraint. Following the path in Hansen et al. (1998) of solving the discrete version to obtain an initial solution for the continuous problem, in the first stage of this heuristic, the SPLP is solved assuming that the demand points are the potential locations for facilities. Then, in the second stage, a fine tuning is performed in the continuous space using Weiszfeld's method. Brimberg and Salhi (2005) introduced zone-dependent fixed costs for the facilities, where they defined zones as polygons. An efficient exact solution algorithm for the single facility case was proposed, whereas, for the multi-facility case, they proposed heuristic procedures.

Drezner et al. (1991) introduced a Weber problem with limited distances. In that problem, the cost for a demand point increases linearly with its distance from the facility until a limit is reached. Afterwards, the cost stays constant at the limiting value. A possible motivation for this problem is that, after a distance limit, the service to demand points may be provided with an alternative method. In that case, the distance limit can be viewed as a break-even point on the cost. In the distance-limited continuous location-allocation problem that we present, as opposed to the constant cost after the distance limit in Drezner et al. (1991), we assume an infinite cost after the distance limit, so our problem is quite different than other distance-limited problems considered in the literature (e.g. in Drezner et al., 2016; 1991; Fernandes et al., 2014).

In our problem, the number of facilities to be opened is a decision variable. For a given number of facilities and without a distance limitation, our problem becomes the MWP, which is NP-hard. We propose a multi-stage heuristic solution method, in which we solve the discrete version of the problem and then adjust facility locations in the continuous space for fine-tuning. The final solution quality highly depends on the initial solution quality we obtain from the discrete version of the problem. Employing the demand points as the only possible locations for the facilities (as is done in Brimberg et al., 2004; Brimberg and Salhi, 2005; and Hansen et al., 1998) would limit the solution quality of the discrete problem. Augmenting this set of possible locations with a small number of additional promising locations is the main idea presented in this paper. Rather than overlaying the area of demand points by a fine grid, as is done in Brimberg and Drezner (2013), we propose 
to solve the PSCP under the distance limitation to obtain these additional locations.

The stages of our algorithm can be described as follows: In the first stage, we solve the PSCP employing the CIPS for the demand points to obtain a set of promising locations to augment the set of demand points. These additional locations provide the minimum set cover for the demand points under the distance limitation. In the second stage, we solve the discrete version of the problem defined over the augmented set. Finally, in the third stage of our heuristic algorithm, starting at the solution of the second stage, we apply Cooper's iterative location-allocation algorithm. Note that, for the location step, we propose a modified version of Weizsfeld's algorithm (Weiszfeld, 1937) to incorporate our coverage distance constraint.

The contributions of this paper can be summarized as follows: We introduce a new problem which has wide applications in the spatial planning of decentralized energy and water distribution systems. Then, we provide the mathematical model of this problem in the continuous space. As the problem is NP-hard, we propose a three-stage heuristic solution algorithm. In order to incorporate the distance limitation constraints, we propose a version of Weizsfeld's algorithm with projections. We conduct computational experiments to illustrate how the proposed algorithm works under different distance limitations and cost parameters for the problem.

The sections of this paper are outlined as follows: A more precise statement and the mathematical formulation of the problem are given in Section 2. Our heuristic solution method for the problem is explained in Section 3. Computational results along with the discussions are provided in Section 4. We conclude our paper in Section 5.

\section{Problem formulation}

Consider a rectangular greenfield of $L \times W$ dimensions with $N$ demand points. The demand point $i$ is at location $\left(a_{i}, b_{i}\right)$ and has an associated weight $w_{i}>0$. Since each demand point is to be served by a single facility, we need at most $N$ facilities to serve all demand points.

Both the electric voltage and the water pressure drop with distance from the source. To prevent from exceeding the maximum allowable drop, there is a limit on the length of each connection. We incorporate this limit in our model by introducing a circular coverage region with the radius $D_{\max }$ around each facility, and assuming that the demand points outside this region cannot be served by the facility. In this paper, we assume that the total demand in each coverage region can be met by a single facility, so we treat the facilities as uncapacitated. Each facility $j$ is located at $\left(x_{j}\right.$, $y_{j}$ ) and has a fixed opening cost of $F$ if serving any demand points.

Our objective is to determine the number and the location of open facilities, and the assignment of demand points to these facilities to minimize the total cost composed of connection (weighted distance) and facility opening costs. Since the facilities are uncapacitated, each demand point will be served by the closest open facility to minimize its connection cost. We assume that all distances are Euclidean. Let us denote the index set $\{1, \ldots, N\}$ by $\mathcal{N}$ and define the decision variables

\footnotetext{
$d_{i j}$ : Euclidean distance between demand point $i \in \mathcal{N}$ and facility $j \in \mathcal{N}$

$\delta_{i}$ : Euclidean distance between demand point $i \in \mathcal{N}$ and closest open facility

$v_{j}= \begin{cases}1, & \text { if facility } j \in \mathcal{N} \text { is open, }\end{cases}$

$\{$, otherwise,

$z_{i j}= \begin{cases}1, & \text { if demand point } i \in \mathcal{N} \text { is served by facility } j \in \mathcal{N}, \\ 0, & \text { otherwise. }\end{cases}$
}

We propose to solve the following mixed integer quadratically constrained programming (MIQCP) problem, denoted by
(DLim-CLAP):

$\min \sum_{j \in \mathcal{N}} v_{j} F+\sum_{i \in \mathcal{N}} w_{i} \delta_{i}$

subject to

$\sum_{j \in \mathcal{N}} z_{i j}=1$

$i \in \mathcal{N}$;

$z_{i j} \leq v_{j}$

$i, j \in \mathcal{N}$

$\delta_{i} \leq D_{\text {max }}$

$i \in \mathcal{N}$

$\delta_{i} \geq \sqrt{L^{2}+W^{2}}\left(z_{i j}-1\right)+d_{i j}$

$i, j \in \mathcal{N}$;

$d_{i j}^{x}=a_{i}-x_{j}$

$i, j \in \mathcal{N}$;

$d_{i j}^{y}=b_{i}-y_{j}$

$i, j \in \mathcal{N}$;

$d_{i j}^{2} \geq\left(d_{i j}^{x}\right)^{2}+\left(d_{i j}^{y}\right)^{2}$

$i, j \in \mathcal{N}$;

$x_{j}, y_{j} \in \mathbb{R}$,

$j \in \mathcal{N}$;

$v_{j} \in\{0,1\}$,

$j \in \mathcal{N}$;

$z_{i j} \in\{0,1\}$,

$i, j \in \mathcal{N}$

$d_{i j}^{x}, d_{i j}^{y} \in \mathbb{R}$,

$i, j \in \mathcal{N}$;

$d_{i j} \geq 0$,

$i, j \in \mathcal{N}$;

$\delta_{i} \geq 0$,

$i \in \mathcal{N}$.

We minimize the total distribution cost in (1) that is composed of facility and connection costs. The constraint set (2) assigns a facility to each demand point. We guarantee by constraints (3) that closed facilities are not assigned to any demand points. The distances of the demand points to their closest facilities are bounded from above by $D_{\max }$ in the constraint set (4). The lower bounds on these distances are presented in constraints (5). Constraints (6) and (7) define the x-coordinate difference $d_{i j}^{x}$ and the y-coordinate difference $d_{i j}^{y}$, respectively, between each demand point $i$ and each facility $j$. Employing these differences, the set of quadratic constraints in (8) define the Euclidean distances between the demand points and the facilities. The decision variables of this optimization problem are defined in (9)-(14).

This optimization problem has $N^{2}+N$ binary and $3 N^{2}+3 N$ continuous decision variables, and $6 N^{2}+3 N$ constraints. For a given number of facilities and without the coverage distance limitations, the DLim-CLAP becomes the MWP which is shown to be NP-hard by Megiddo and Supowit (1984). In the next section, we propose a three-stage heuristic method for the solution of the DLim-CLAP.

\section{A three-stage heuristic algorithm}

We follow the steps of Hansen et al. (1998), where a heuristic method to solve the MWP was proposed. The discrete counterpart of the MWP is the well studied p-median problem where the facility locations are chosen from a given set of candidate locations. While the p-median problem is also an NP-hard problem (Kariv and Hakimi, 1979), solving a p-median problem exactly is a lot easier than solving a MWP as discussed by Hansen et al. (1998). In addition, it was also observed in Hansen et al. (1998) that some of the optimal facility locations in the MWP coincide with the demand locations. Motivated by these observations, Hansen et al. (1998) proposed a heuristic solution method for the MWP. This method first solves the p-median problem where the candidate locations for the facilities are the demand locations. Then, a Weber problem (the problem in Weber, 1929 of finding a point minimizing the sum of weighted distances from given points) is solved for each cluster of demand points served by the same facility. In 


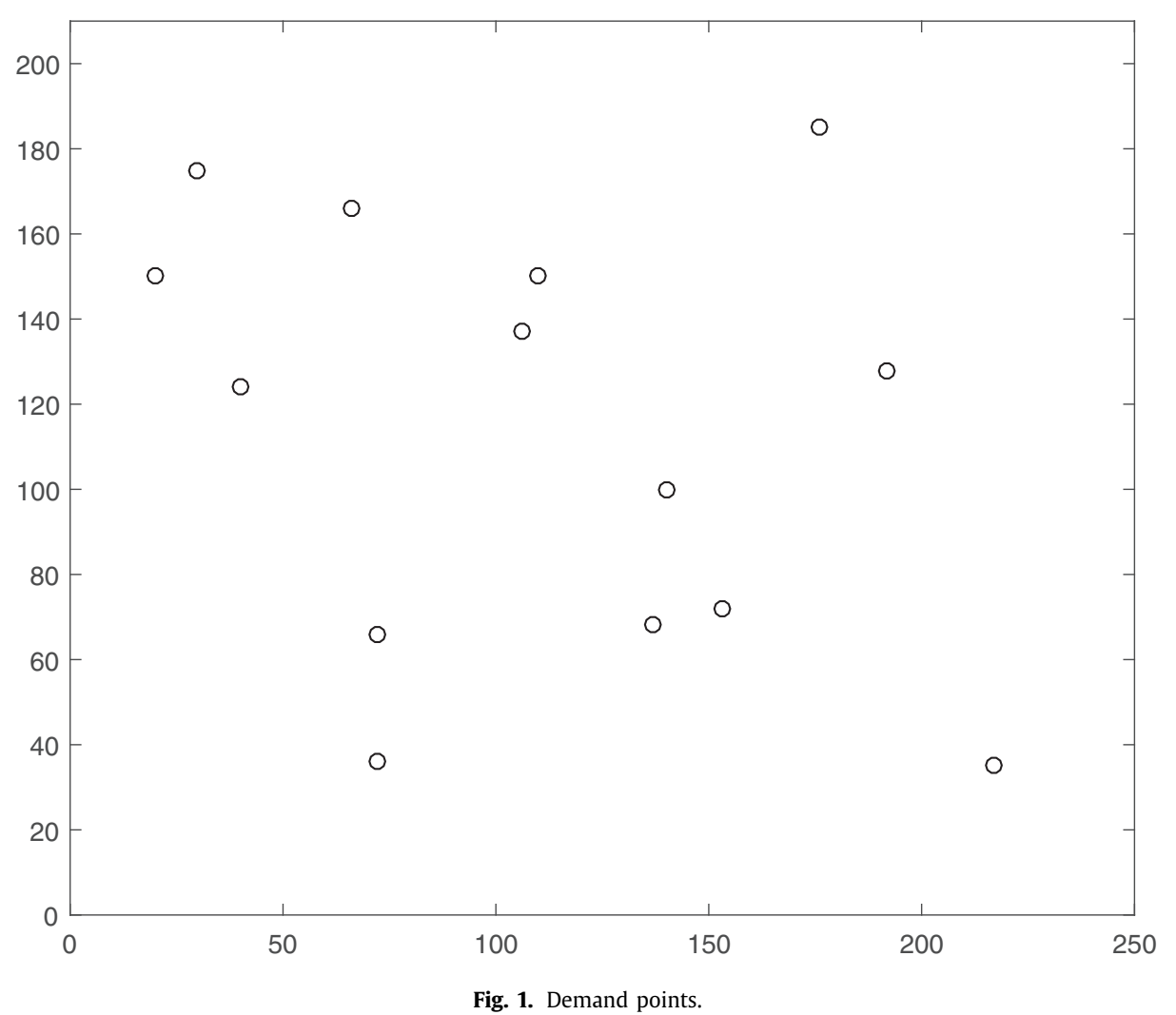

this study, we adopt a similar approach and propose a three-stage heuristic method to solve the DLim-CLAP.

In order to illustrate our solution method graphically, we present the running example with 14 demand points shown in Fig. 1. In this example, the facility cost is given as $F=1000$, the coverage distance is given as $D_{\max }=30$, and the weights are given as $w_{i}=17$ for all $i \in\{1, \ldots, 14\}$.

In the first stage of our method, we determine the minimum number of facilities and their locations to cover all demand points under the given coverage distance. In other words, in this stage, we are solving the DLim-CLAP problem with $w_{i}=0$ for all demand points $i$.

\subsection{Stage 1: solving the PSCP}

In order to solve the PSCP defined for our coverage distance, we first determine the intersection points of all circles centered at the demand points and with the radius $D_{\max }$. These points are suggested by Church (1984) to be used to find an optimal solution to the PSCP by solving a SCP. We show these points for our running example in Fig. 2. Note that if the circle of a demand point does not intersect with any other circle, then the center of the circle, the demand point itself, is included in the set of the circle intersection points that we denote by $\mathcal{C}$ (see the demand point in the lower right corner of Fig. 2). Let us denote the cardinality of this set by $C$, which is equal to 35 in the running example. Then, we determine the coverage region for each point in the set of circle intersection points as in Fig. 3.

The demand points in the coverage region of each circle intersection point form a set. Considering the collection of all these sets, we formulate and solve the following set covering problem: For each demand point $i \in \mathcal{N}$ and for each circle intersection point $k \in \mathcal{C}$, let us define the coverage parameter

$\alpha_{i k}= \begin{cases}1, & \text { if } d_{i k} \leq D_{\max } \\ 0, & \text { otherwise }\end{cases}$
In this formulation, $d_{i k}$ denotes the Euclidean distance between the demand point $i$ and the circle intersection point $k$. Then, we solve the unicost (SCP) defined as

$\min \sum_{k \in \mathcal{C}} v_{k}$

subject to

$\begin{array}{ll}\sum_{k \in \mathcal{C}} \alpha_{i k} v_{k} \geq 1, & i \in \mathcal{N} ; \\ v_{k} \in\{0,1\}, & k \in \mathcal{C} .\end{array}$

The objective value of the optimal solution will yield the minimum number of facilities needed. The PSCP solution (with six facilities) for our running example is shown in Fig. 4. Once we obtain the locations of the minimum number of covering facilities $\left(v_{k}^{*}=1\right)$, we conclude the first stage of our heuristic.

\subsection{Stage 2: determining the number of facilities}

In the second stage, we determine the number of facilities by solving the discrete version of the DLim-CLAP, which we call distance-limited "plant" location problem, DLim-PLP, to be consistent with the literature. Rather than limiting the candidate locations for the facilities to the demand locations as in Brimberg et al. (2004), Brimberg and Salhi (2005) and Hansen et al. (1998), we augment the set of demand locations with the locations obtained in the first stage to form the candidate locations for the facilities. Let us denote this augmented set of candidate locations by $\mathcal{M}$ and its cardinality by $M$, which is equal to 19 in the running example. The candidate facility locations in our running example are shown in Fig. 5, where the circle intersection points in the PSCP solution are indicated by the diamonds and the demand points are indicated by the circles. Note that there exists a demand point in the lower right corner of this figure that is also a circle intersection point. 


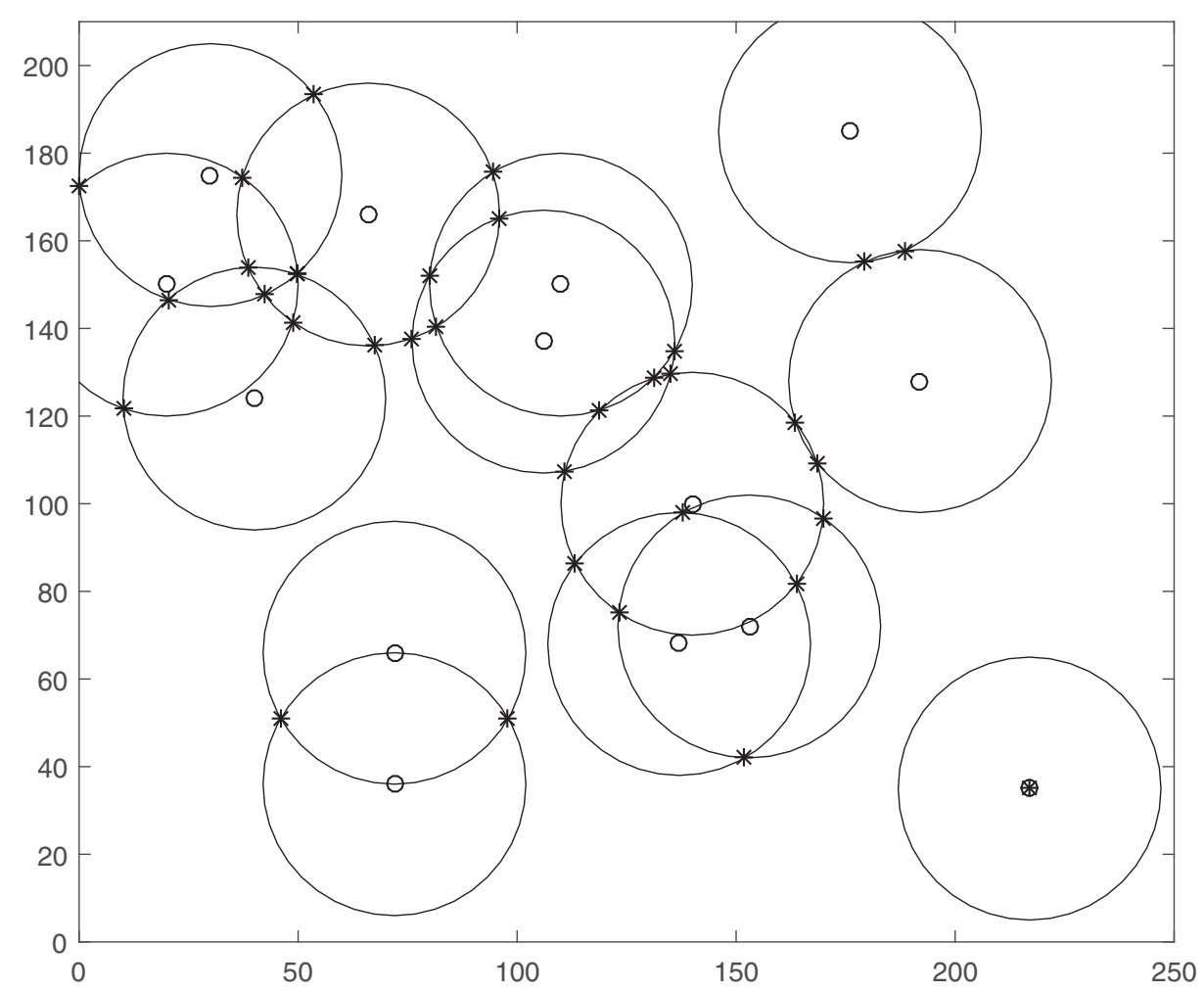

Fig. 2. Circle intersection points.

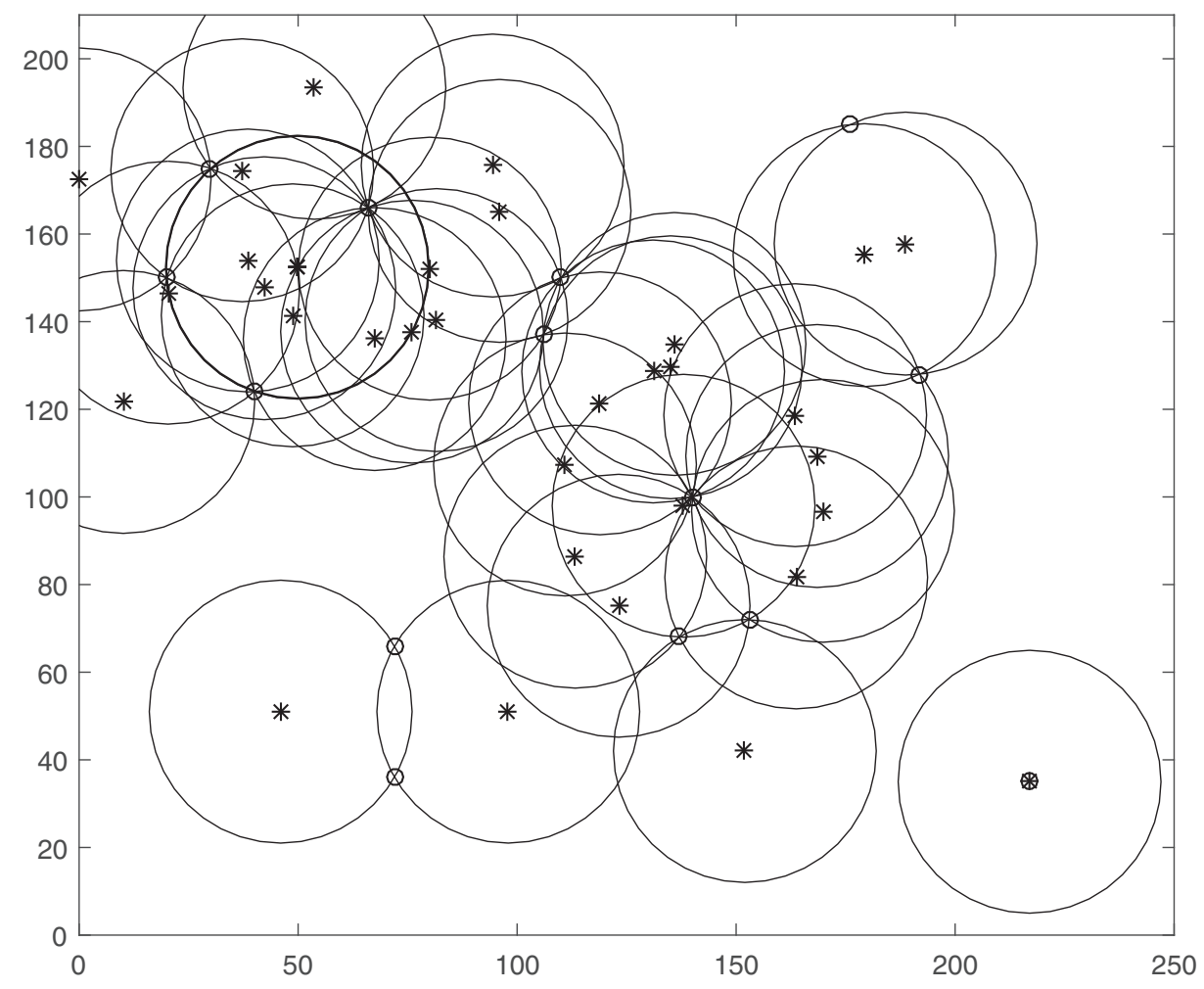

Fig. 3. Coverage regions for circle intersection points.

Since we provide additional candidate locations to the DLimPLP, the solution time is expected to increase but in return we may obtain a better solution. Our computational results show that the PSCP solution provides a reasonable number of additional candidate locations that improve performance considerably in several instances without a major increase in the solution times.
We formulate and solve the DLim-PLP (i.e, the discrete version of the DLim-CLAP) as follows:

$\min \sum_{j \in \mathcal{M}} v_{j} F+\sum_{i \in \mathcal{N}} \sum_{j \in \mathcal{M}} z_{i j} w_{i} d_{i j}$ 


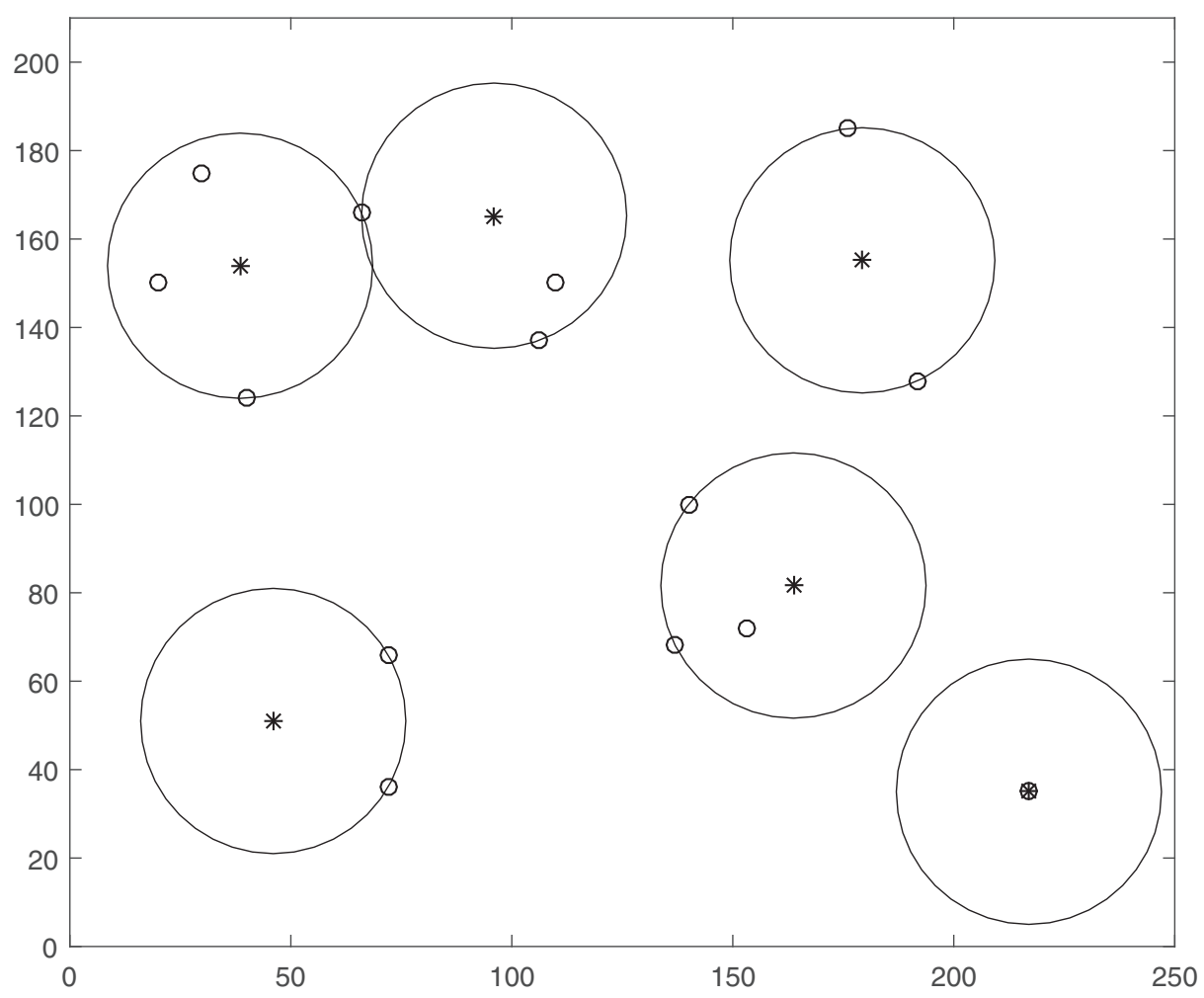

Fig. 4. The PSCP solution.

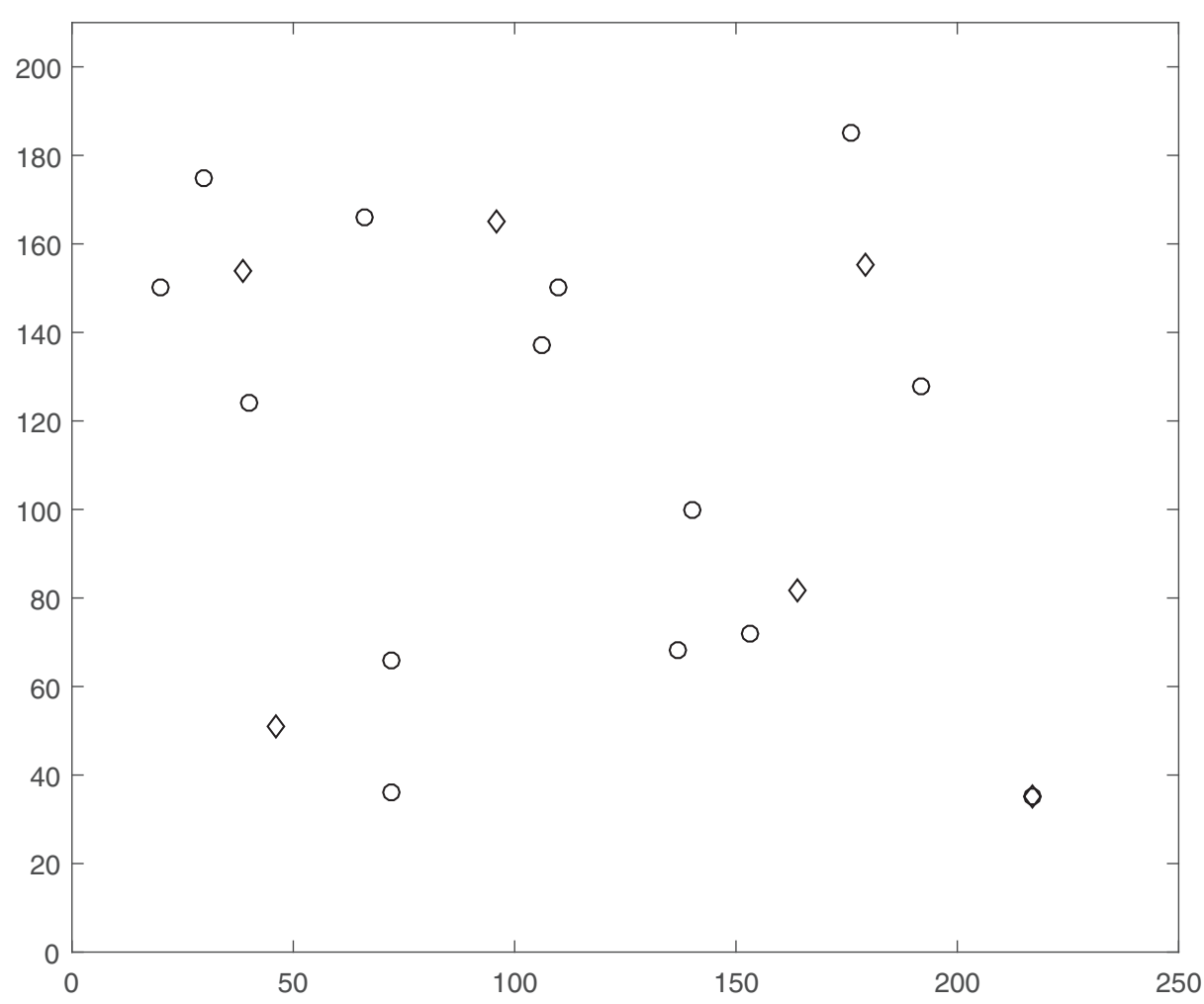

Fig. 5. Possible facility locations.

subject to

$\sum_{j \in \mathcal{M}} z_{i j}=1$,

$z_{i j} \leq v_{j}$,

$\sum_{j \in \mathcal{M}} z_{i j} d_{i j} \leq D_{\max }$

$$
v_{j} \in\{0,1\}
$$$$
j \in \mathcal{M}
$$$$
i \in \mathcal{N}
$$$$
i \in \mathcal{N}, j \in \mathcal{M}
$$

$z_{i j} \in\{0,1\}$,

$$
i \in \mathcal{N}, j \in \mathcal{M} \text {. }
$$

In this formulation, $d_{i j}$ indicates the Euclidean distance between the demand point $i$ and the candidate location $j$. Since the distances are no longer decision variables, the objective function in 


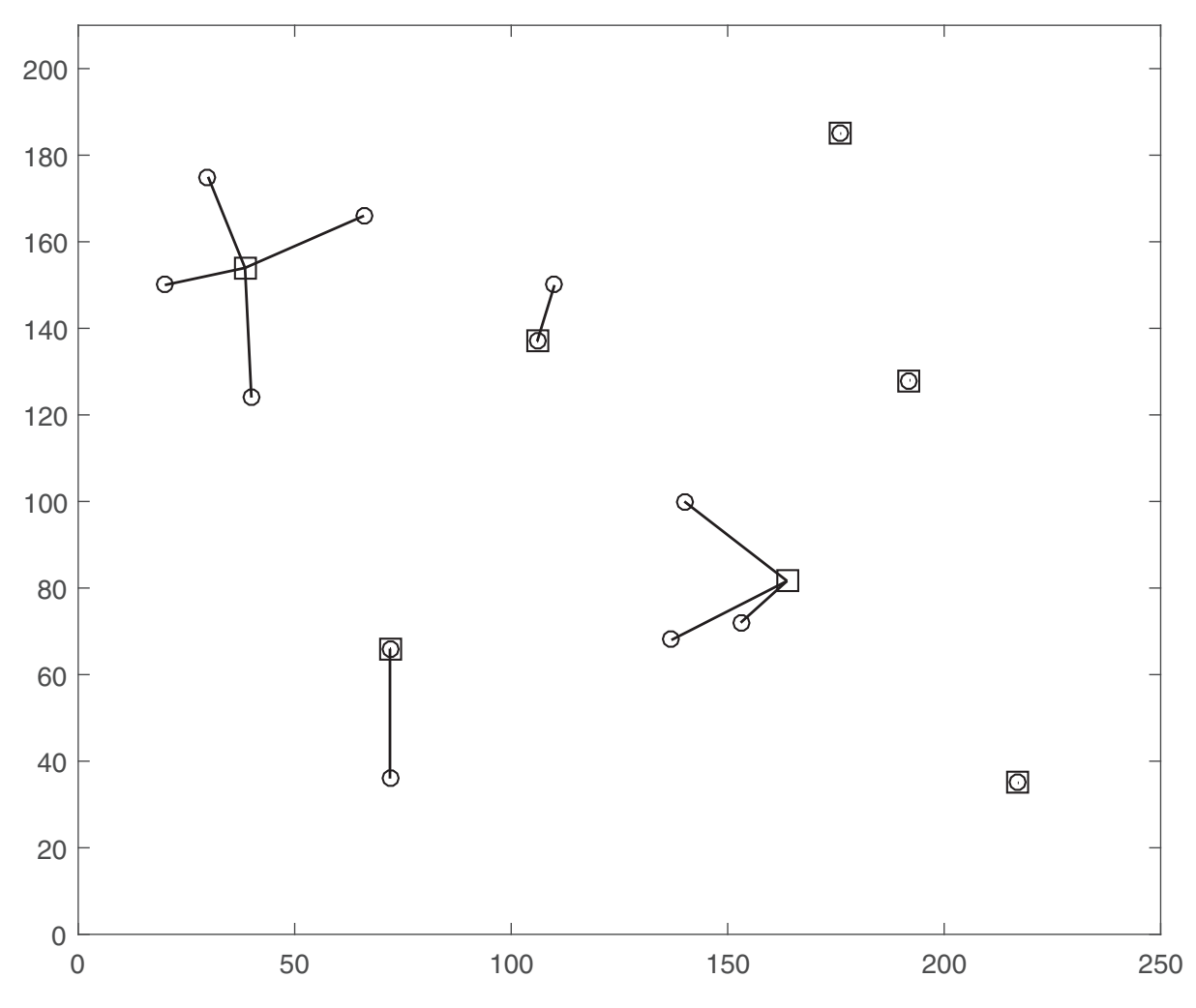

Fig. 6. DLim-PLP solution.

(15) is linear. The constraints (16) and (17) are the constraints (2) and (3), respectively, rewritten for the augmented candidate location set. The constraints (18) follow from the constraints (4). We define the facility opening and the assignment decision variables in (19) and (20). Note that Krysta and Solis-Oba. (2001) and Weng (2013) have similar formulations for the problem without any weights associated with the demand points.

The solution of this model yields the number of facilities $V=\sum_{j \in \mathcal{M}} v_{j}$ and the assignments $z_{i j}$ of these facilities to the demand points. The solution for our running example with seven facilities is presented in Fig. 6, where the locations of the facilities are shown by the squares. Note that there are five facilities in this figure that are co-located with the demand points. The rest of the demand points are connected to the facilities in a star topology. Note also that the two demand points in the upper right corner can be served by a single facility as in Fig. 4. However, the distance between these two demand points times the minimum weight among them exceeds the cost of a facility. Therefore, a second facility is opened and both facilities are co-located with these demand points.

The second stage of the heuristic method results with a number of facilities, some co-located with the demand points and others possibly at the circle intersection points, and the assignments of these facilities to the demand points. Let us denote the cluster of demand points for each facility $k$ by $C_{k}$ defined as

$C_{k}=\left\{i \mid z_{i k}=1\right\}$

for $k \in\{1, \ldots, V\}$. Next, we adjust the facility locations in the continuous space to decrease the total cost.

\subsection{Stage 3: determining the facility locations in the continuous space}

In the third stage, starting with the facility locations obtained in the second stage, we apply Cooper's alternating location and allocation algorithm described in Cooper (1964). This algorithm iteratively re-allocates demand points to the closest facilities so that clusters are updated and then relocates the facility for each cluster to minimize the weighted distance cost from each cluster, until no changes are observed in the demand point allocations and the facility locations.

At each location step of Cooper's algorithm, we solve the optimization problem below, denoted by DLim-Geom, to find the location of the facility for each cluster $C_{k}$ :

$\min \sum_{i \in C_{k}} w_{i} d_{i k}$

subject to

$d_{i k} \leq D_{\max }, \quad i \in C_{k}$;

$d_{i k}^{x}=a_{i}-x_{k}, \quad i \in C_{k}$;

$d_{i k}^{y}=b_{i}-y_{k}, \quad i \in C_{k}$;

$d_{i k}^{2} \geq\left(d_{i k}^{x}\right)^{2}+\left(d_{i k}^{y}\right)^{2}, \quad i \in C_{k} ;$

$x_{k}, y_{k} \in \mathbb{R}$,

$d_{i k}^{x}, d_{i k}^{y} \in \mathbb{R}$,

$i \in C_{k}$;

$d_{i k} \geq 0$,

$i \in C_{k}$.

Constraints (21)-(24) and the variable definitions (25)-(27) are the constraints (4), (6)-(8) and the variable definitions (9), (12), (13), respectively, written for the cluster $C_{k}$. Without the set of constraints in (21), the DLim-Geom reduces to the Weber problem in Weber (1929), which aims to find a point that minimizes the sum of the weighted distances from the points within the cluster. Vardi and Zhang proposed a modified Weiszfeld algorithm in 


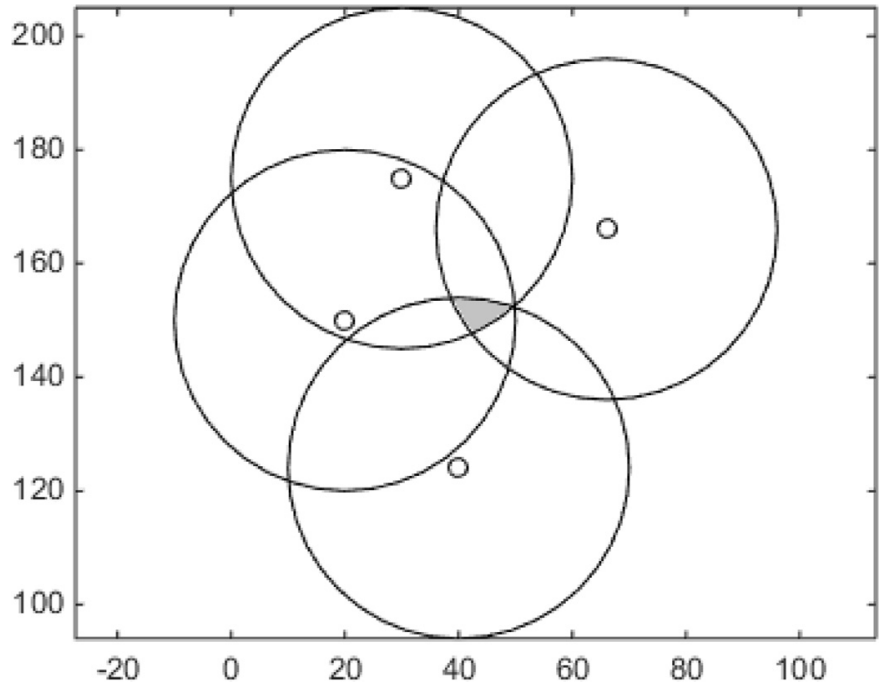

Fig. 7. Feasible region for the facility.

Vardi and Zhang (2001) for the Weber problem. The constraints (21) limit the feasible region for the facility location as in Fig. 7, where we zoom in to the cluster in the upper left corner of Fig. 6. In this figure, the facility that serves the four demand points (indicated by the little circles) has to be located within the gray area to satisfy the constraints (21). Since the feasible region for the facility location is the intersection of overlapping circles, it is always convex. The algorithm proposed by Vardi and Zhang may locate the facility outside this region, i.e., it may return an infeasible solution for the DLim-Geom. Next, we present an iterative heuristic method with projections to solve the DLim-Geom.

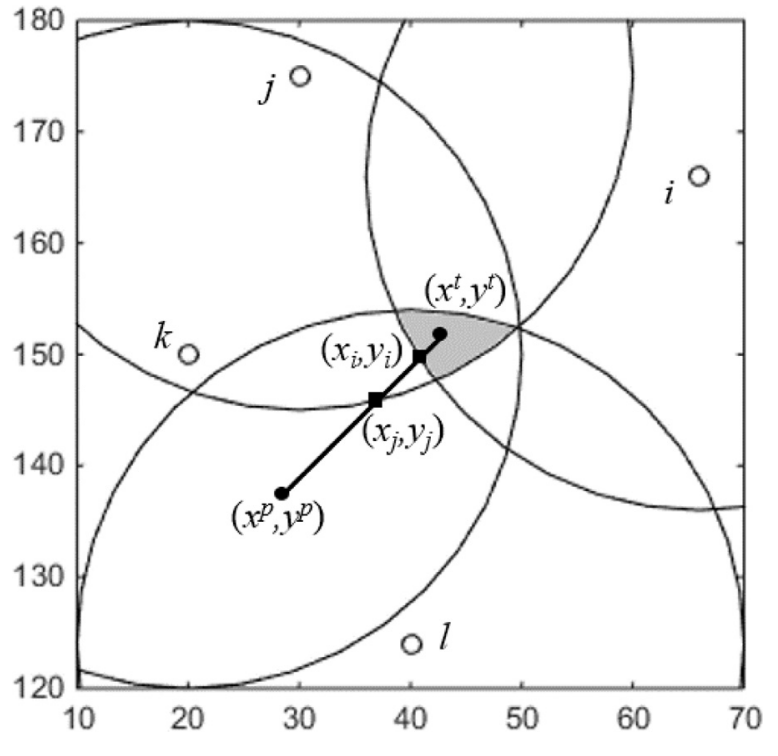

Fig. 8. Projection.

\subsubsection{An iterative heuristic method with projections}

Let $\left(x^{0}, y^{0}\right)$ and $r$ be the center and the radius, respectively, of the minimum circle enclosing all points in the cluster $C_{k}$. In the following discussion, we assume that $r \leq D_{\max }$, otherwise the DLim-Geom would be infeasible. We start our algorithm at $\left(x^{0}, y^{0}\right)$, which is a guaranteed feasible location for the DLim-Geom, as all demand points are within a $D_{\max }$ distance from this location.

Our modification to the iterative algorithm by Vardi and Zhang is to project each proposed location to the convex feasible set of the DLim-Geom at every iteration, so that feasibility is always preserved. Let $\left(x^{t}, y^{t}\right)$ be the location at iteration $t$. We assume that

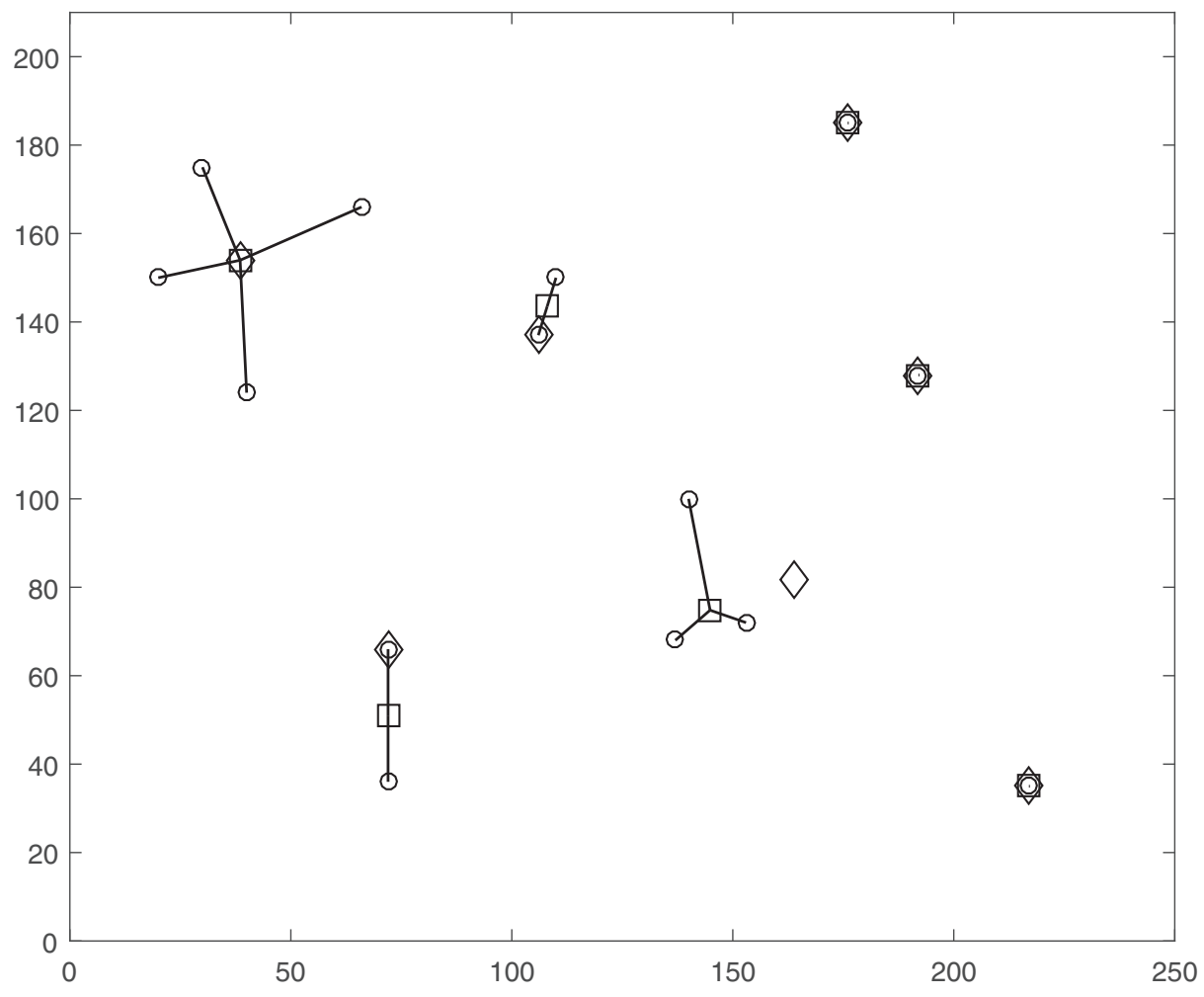

Fig. 9. Final solution. 


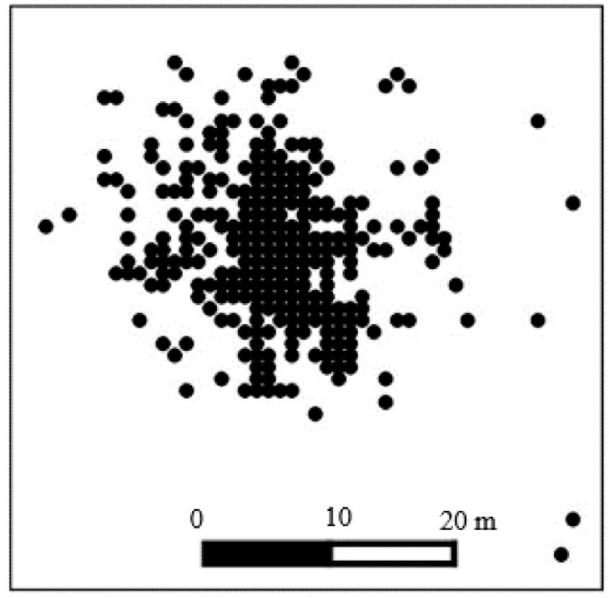

a) W287

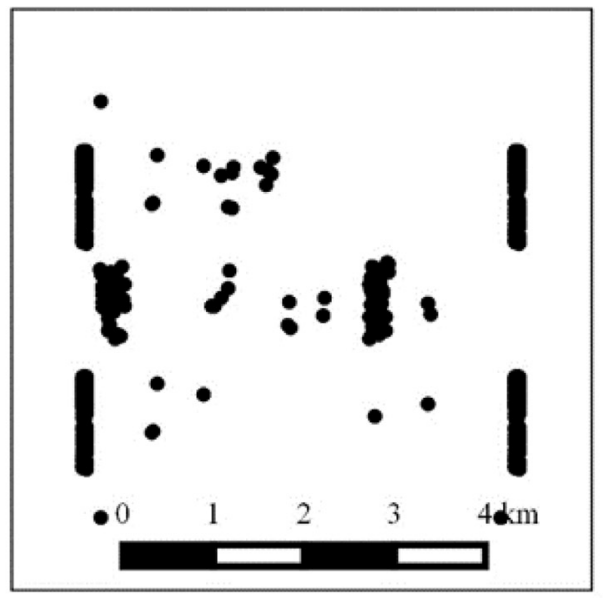

b) U654

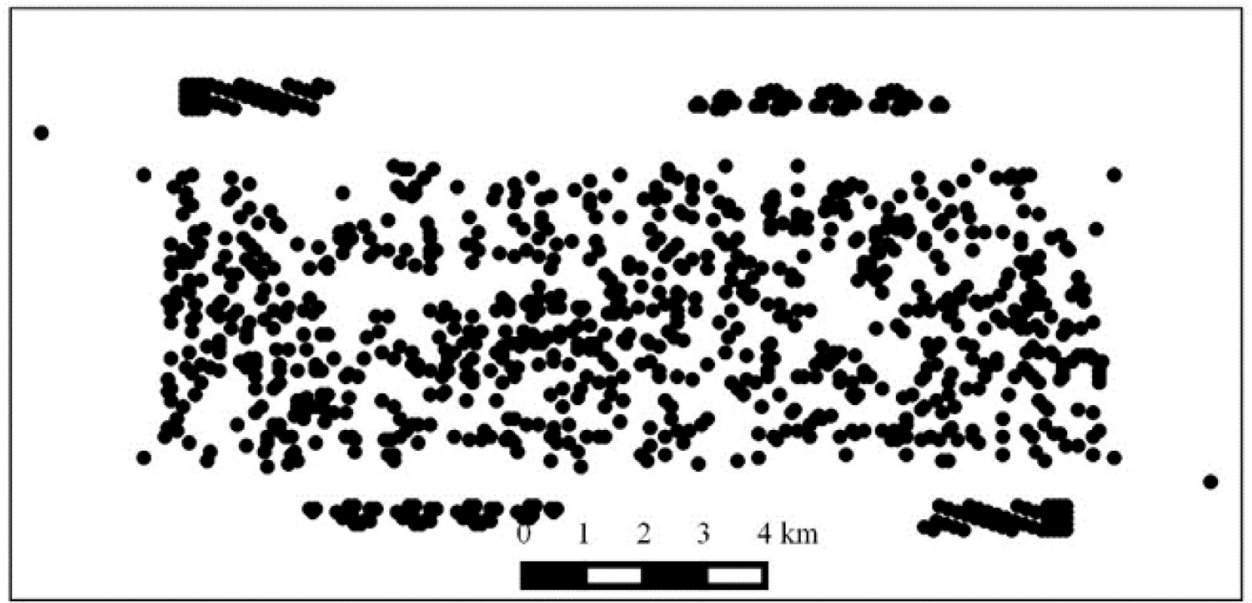

c) $\cup 1060$

Fig. 10. Demand locations in example data sets.

this location is feasible for the DLim-Geom as we start from a feasible location (center of the minimum enclosing circle) and apply the projection at every iteration to preserve feasibility. Let $\left(x^{p}, y^{p}\right)$ be the location proposed by Vardi and Zhang's algorithm for the next iteration (see Vardi and Zhang, 2001 for details on how a new location is proposed). If the proposed location is also feasible, i.e., if the constraints (21) are already satisfied by $\left(x^{p}, y^{p}\right)$ for all demand points in the cluster, then we accept it as the location for the next iteration so that $\left(x^{t+1}, y^{t+1}\right)=\left(x^{p}, y^{p}\right)$. If, on the other hand, the proposed location is not feasible, i.e., if there are demand points that are more than $D_{\max }$ away from $\left(x^{p}, y^{p}\right)$, then we project the facility's location onto the feasible region as follows: Consider the line segment between $\left(x^{t}, y^{t}\right)$ and $\left(x^{p}, y^{p}\right)$. Our projection method locates facility at the intersection point of this line segment and the boundary of the feasible region. Let $A \subset C_{k}$ denote the set of demand points whose distances to the proposed location $\left(x^{p}, y^{p}\right)$ exceeds $D_{\text {max }}$. For each demand point $\left(a_{i}, b_{i}\right)$ in $A$, we determine the location $\left(x_{i}, y_{i}\right)$ that is both $D_{\max }$ away from the demand point and on the line segment whose end points are $\left(x^{t}, y^{t}\right)$ and $\left(x^{p}, y^{p}\right)$ (see $\left(x_{i}, y_{i}\right)$ and $\left(x_{j}, y_{j}\right)$ in Fig. 8 associated with the demand points $i$ and $j$, respectively). As the points on the line segment can be described by the equation $\left(x^{t}, y^{t}\right)+\beta\left(x^{p}-x^{t}, y^{p}-y^{t}\right)$ for $\beta \in[0,1]$, we determine the corresponding $\beta_{i} \in[0,1]$ for $\left(x_{i}, y_{i}\right)$ as the solution to the second order polynomial equation:

$\left(x^{t}+\beta_{i}\left(x^{p}-x^{t}\right)-a_{i}\right)^{2}+\left(y^{t}+\beta_{i}\left(y^{p}-y^{t}\right)-b_{i}\right)^{2}=D_{\max }^{2}$

for all $i \in A$.
Since the circular regions are convex, for demand points that are in $C_{k} \backslash A$, all points on the line segment are within the $D_{\max }$ distance. For demand points $i \in A$, however, only the points on the line segment with $\beta \in\left[0, \beta_{i}\right]$ are within the $D_{\max }$ distance. Therefore, we determine $\beta_{\min }=\min _{i \in A} \beta_{i}$ and set the facility location for the next iteration as

$\left(x^{(t+1)}, y^{(t+1)}\right)=\left(x^{t}, y^{t}\right)+\beta_{\min }\left(x^{p}-x^{t}, y^{p}-y^{t}\right)$

This is the point of intersection of the line segment between $\left(x^{t}, y^{t}\right)$ and $\left(x^{p}, y^{p}\right)$, and the boundary of the feasible region. Since we project locations outside the feasible region onto the boundary of the feasible region, we preserve feasibility at each iteration.

We repeat the location updates until the decrease in the objective value falls below an $\epsilon$ threshold. Since the objective value cannot decrease forever with an amount larger than $\epsilon$, the algorithm stops in a number of iterations that depends on the $\epsilon$ value.

Applying the third stage on our running example, we obtain the solution shown in Fig. 9 with a cost of 10,228. The diamonds and the squares show the locations of the facilities at the beginning (as in Fig. 6) and at the end of the third stage, respectively. If we solve the DLim-CLAP for this small example under a time limit of ten hours, we obtain the same solution as the best feasible solution with an optimality gap of $12 \%$. 
Table 1

The PSCP solutions.

\begin{tabular}{lllll}
\hline Data & $D_{\max }$ & $\mathrm{C}$ & Solution & Time \\
\hline W287 & 5 & 34,025 & 14 & 1 \\
& 10 & 71,642 & 6 & 25 \\
& 15 & 80,516 & 3 & 22 \\
& 20 & 81,719 & 2 & 27 \\
& 25 & 82,048 & 2 & 27 \\
U654 & 200 & 50,905 & 36 & 1 \\
& 400 & 74,520 & 17 & 2 \\
& 600 & 86,969 & 13 & 5 \\
& 800 & 97,854 & 9 & 3 \\
U1060 & 1000 & 126,614 & 7 & 7 \\
& 200 & 8016 & 301 & 0 \\
& 400 & 22,645 & 127 & 13 \\
& 600 & 45,104 & 73 & 28 \\
& 800 & 75,651 & 50 & 180 \\
& 1000 & 111,934 & 35 & 102 \\
\hline
\end{tabular}

\section{Computational results}

In this section, we perform experiments on three sets of data that are widely used for studying the MWP (Brimberg et al., 2008). These data sets include the 287 node example from Bongartz et al. (1994), and 654 and 1060 customer problems from the TSP library (Reinelt, 1991). We denote these sets by W287, U654, and U1060, and present their demand locations in Fig. 10. W287 has 287 demand points with weights $w_{i}$ ranging between 1 and 698. U654 and U1060, on the other hand, have 654 and 1060 demand points, respectively, each with a unit weight $w_{i}=1$. These data sets are also used in Brimberg et al. (2004) for the multi-source Weber problem with constant opening costs.

In our experiments, the cost of deploying a facility $F$ takes values from $\{50,100,200,500,5000\},\{1000,2000,5000$, $10,000,15,000\}$, and $\{1000,2000,5000,10,000,15,000\}$ for data sets W287, U654, and U1060, respectively. Distance limits $D_{\max }$ are selected from $\{5,10,15,20,25\}$ for W287 and from $\{200,400$,

Table 2

Comparison of methods on W287 and U654.

\begin{tabular}{|c|c|c|c|c|c|c|c|c|c|c|c|c|}
\hline \multirow[b]{2}{*}{ Data } & \multirow[b]{2}{*}{$F$} & \multirow[b]{2}{*}{$D_{\max }$} & \multicolumn{4}{|c|}{ w/o PSCP } & \multicolumn{4}{|c|}{ w/ PSCP } & \multicolumn{2}{|c|}{ \% Cost Diff. } \\
\hline & & & $V$ & $2 \mathrm{~S}$ Cost & 2S Time & 3S Cost & $V$ & $2 \mathrm{~S}$ Cost & 2S Time & 3S Cost & $2 \mathrm{~S}$ & $3 \mathrm{~S}$ \\
\hline \multirow[t]{5}{*}{ W287 } & \multirow[t]{5}{*}{50} & 5 & 53 & 4157 & 1 & 4157 & 50 & 4055 & 1 & 4033 & $2.45 \%$ & $2.98 \%$ \\
\hline & & 10 & 46 & 3951 & 8 & 3951 & 45 & 3926 & 10 & 3926 & $0.63 \%$ & $0.63 \%$ \\
\hline & & 15 & 44 & 3890 & 11 & 3889 & 44 & 3890 & 16 & 3889 & $0.00 \%$ & $0.00 \%$ \\
\hline & & 20 & 44 & 3890 & 14 & 3889 & 44 & 3890 & 17 & 3889 & $0.00 \%$ & $0.00 \%$ \\
\hline & & 25 & 43 & 3885 & 16 & 3884 & 43 & 3885 & 18 & 3884 & $0.00 \%$ & $0.00 \%$ \\
\hline \multirow[t]{5}{*}{ W287 } & \multirow[t]{5}{*}{100} & 5 & 42 & 6511 & 1 & 6499 & 39 & 6285 & 1 & 6272 & $3.48 \%$ & $3.49 \%$ \\
\hline & & 10 & 34 & 5932 & 11 & 5932 & 33 & 5857 & 8 & 5857 & $1.27 \%$ & $1.27 \%$ \\
\hline & & 15 & 32 & 5770 & 12 & 5770 & 32 & 5770 & 15 & 5770 & $0.00 \%$ & $0.00 \%$ \\
\hline & & 20 & 32 & 5770 & 17 & 5770 & 32 & 5770 & 20 & 5770 & $0.00 \%$ & $0.00 \%$ \\
\hline & & 25 & 31 & 5717 & 18 & 5717 & 31 & 5717 & 29 & 5717 & $0.00 \%$ & $0.00 \%$ \\
\hline \multirow[t]{5}{*}{ W287 } & \multirow[t]{5}{*}{200} & 5 & 32 & 10,236 & 1 & 10,220 & 29 & 9643 & 1 & 9632 & $5.80 \%$ & $5.75 \%$ \\
\hline & & 10 & 23 & 8746 & 8 & 8741 & 22 & 8621 & 12 & 8619 & $1.43 \%$ & $1.39 \%$ \\
\hline & & 15 & 20 & 8359 & 18 & 8357 & 20 & 8359 & 21 & 8357 & $0.00 \%$ & $0.00 \%$ \\
\hline & & 20 & 20 & 8313 & 22 & 8305 & 20 & 8313 & 24 & 8305 & $0.00 \%$ & $0.00 \%$ \\
\hline & & 25 & 19 & 8171 & 21 & 8157 & 19 & 8171 & 23 & 8157 & $0.00 \%$ & $0.00 \%$ \\
\hline \multirow[t]{5}{*}{ W287 } & \multirow[t]{5}{*}{500} & 5 & 23 & 17,964 & 1 & 17,905 & 18 & 16,026 & 1 & 16,004 & $10.79 \%$ & $10.62 \%$ \\
\hline & & 10 & 13 & 13,609 & 16 & 13,609 & 12 & 13,228 & 24 & 13,228 & $2.80 \%$ & $2.80 \%$ \\
\hline & & 15 & 10 & 12,312 & 28 & 12,290 & 10 & 12,312 & 36 & 12,290 & $0.00 \%$ & $0.00 \%$ \\
\hline & & 20 & 9 & 12,134 & 28 & 12,113 & 9 & 12,060 & 30 & 12,024 & $0.61 \%$ & $0.74 \%$ \\
\hline & & 25 & 8 & 11,635 & 23 & 11,635 & 8 & 11,635 & 26 & 11,635 & $0.00 \%$ & $0.00 \%$ \\
\hline \multirow{5}{*}{ W287 } & \multirow{5}{*}{$5 \mathrm{~K}$} & 5 & 19 & 106,815 & 2 & 106,008 & 14 & 83,889 & 1 & 82,867 & $21.46 \%$ & $21.83 \%$ \\
\hline & & 10 & 8 & 53,214 & 38 & 52,903 & 7 & 48,453 & 32 & 48,453 & $8.95 \%$ & $8.41 \%$ \\
\hline & & 15 & 4 & 35,713 & 35 & 35,670 & 4 & 35,665 & 37 & 35,603 & $0.13 \%$ & $0.19 \%$ \\
\hline & & 20 & 3 & 31,455 & 54 & 31,217 & 2 & 27,618 & 58 & 27,555 & $12.20 \%$ & $11.73 \%$ \\
\hline & & 25 & 2 & 26,837 & 41 & 26,837 & 2 & 26,837 & 43 & 26,837 & $0.00 \%$ & $0.00 \%$ \\
\hline \multirow[t]{5}{*}{ U654 } & $1 \mathrm{~K}$ & 200 & 47 & 81,100 & 11 & 80,545 & 44 & 79,485 & 18 & 78,661 & $1.99 \%$ & $2.34 \%$ \\
\hline & & 400 & 41 & 77,453 & 17 & 77,099 & 36 & 76,403 & 9 & 75,292 & $1.36 \%$ & $2.34 \%$ \\
\hline & & 600 & 33 & 75,131 & 12 & 74,687 & 33 & 75,131 & 16 & 74,686 & $0.00 \%$ & $0.00 \%$ \\
\hline & & 800 & 32 & 74,408 & 19 & 73,968 & 32 & 74,408 & 21 & 73,968 & $0.00 \%$ & $0.00 \%$ \\
\hline & & 1000 & 32 & 74,408 & 17 & 73,968 & 32 & 74,408 & 18 & 73,968 & $0.00 \%$ & $0.00 \%$ \\
\hline U654 & $2 \mathrm{~K}$ & 200 & 43 & 125,833 & 13 & 125,297 & 40 & 121,218 & 24 & 120,412 & $3.67 \%$ & $3.90 \%$ \\
\hline & & 400 & 36 & 115,774 & 30 & 115,451 & 30 & 109,233 & 11 & 108,167 & $5.65 \%$ & $6.31 \%$ \\
\hline & & 600 & 27 & 104,678 & 19 & 103,794 & 27 & 104,678 & 25 & 103,794 & $0.00 \%$ & $0.00 \%$ \\
\hline & & 800 & 25 & 103,328 & 22 & 102,660 & 25 & 103,328 & 17 & 102,660 & $0.00 \%$ & $0.00 \%$ \\
\hline & & 1000 & 24 & 103,063 & 18 & 102,246 & 24 & 103,016 & 18 & 102,246 & $0.05 \%$ & $0.00 \%$ \\
\hline U654 & $5 \mathrm{~K}$ & 200 & 39 & 252,164 & 19 & 251,681 & 36 & 238,549 & 22 & 237,796 & $5.40 \%$ & $5.52 \%$ \\
\hline & & 400 & 28 & 212,407 & 23 & 212,154 & 21 & 185,612 & 26 & 184,653 & $12.62 \%$ & $12.96 \%$ \\
\hline & & 600 & 19 & 171,390 & 33 & 170,441 & 19 & 170,729 & 35 & 169,336 & $0.39 \%$ & $0.65 \%$ \\
\hline & & 800 & 15 & 163,722 & 39 & 163,174 & 14 & 163,194 & 43 & 161,454 & $0.32 \%$ & $1.05 \%$ \\
\hline & & 1000 & 13 & 156,104 & 22 & 155,456 & 13 & 156,097 & 20 & 155,456 & $0.00 \%$ & $0.00 \%$ \\
\hline U654 & $10 \mathrm{~K}$ & 200 & 39 & 447,164 & 11 & 446,681 & 36 & 418,549 & 20 & 417,796 & $6.40 \%$ & $6.47 \%$ \\
\hline & & 400 & 28 & 352,407 & 17 & 352,154 & 20 & 285,793 & 27 & 284,128 & $18.90 \%$ & $19.32 \%$ \\
\hline & & 600 & 17 & 256,687 & 39 & 255,742 & 16 & 252,261 & 52 & 249,352 & $1.72 \%$ & $2.50 \%$ \\
\hline & & 800 & 15 & 238,722 & 47 & 238,174 & 13 & 232,923 & 50 & 229,700 & $2.43 \%$ & $3.56 \%$ \\
\hline & & 1000 & 12 & 220,776 & 24 & 219,752 & 12 & 220,776 & 28 & 219,752 & $0.00 \%$ & $0.00 \%$ \\
\hline U654 & $15 \mathrm{~K}$ & 200 & 39 & 642,164 & 11 & 641,681 & 36 & 598,549 & 22 & 597,796 & $6.79 \%$ & $6.84 \%$ \\
\hline & & 400 & 28 & 492,407 & 17 & 492,154 & 18 & 382,015 & 62 & 378,753 & $22.42 \%$ & $23.04 \%$ \\
\hline & & 600 & 16 & 337,128 & 44 & 336,046 & 15 & 327,703 & 58 & 324,656 & $2.80 \%$ & $3.39 \%$ \\
\hline & & 800 & 15 & 313,722 & 61 & 313,174 & 12 & 295,492 & 82 & 295,492 & $5.81 \%$ & $5.65 \%$ \\
\hline & & 1000 & 11 & 280,637 & 28 & 279,641 & 11 & 280,637 & 24 & 279,641 & $0.00 \%$ & $0.00 \%$ \\
\hline
\end{tabular}


Table 3

Comparison of methods on U1060.

\begin{tabular}{|c|c|c|c|c|c|c|c|c|c|c|c|}
\hline \multirow[b]{2}{*}{$F$} & \multirow[b]{2}{*}{$D_{\max }$} & \multicolumn{4}{|c|}{ w/o PSCP } & \multicolumn{4}{|c|}{ w/ PSCP } & \multicolumn{2}{|c|}{ \% Cost Diff. } \\
\hline & & $V$ & 2S Cost & 2S Time & 3S Cost & V & $2 \mathrm{~S}$ Cost & 2S Time & $3 \mathrm{~S}$ Cost & $2 S$ & $3 S$ \\
\hline \multirow[t]{5}{*}{$1 \mathrm{~K}$} & 200 & 481 & 553,562 & 29 & 552,619 & 302 & 447,350 & 14 & 434,834 & $19.19 \%$ & $21.31 \%$ \\
\hline & 400 & 198 & 376,053 & 4 & 373,288 & 186 & 373,462 & 5 & 370,294 & $0.69 \%$ & $0.80 \%$ \\
\hline & 600 & 160 & 365,149 & 5 & 363,314 & 160 & 365,056 & 5 & 363,270 & $0.03 \%$ & $0.01 \%$ \\
\hline & 800 & 153 & 363,597 & 5 & 362,127 & 153 & 363,597 & 5 & 362,127 & $0.00 \%$ & $0.00 \%$ \\
\hline & 1000 & 153 & 363,596 & 8 & 362,120 & 153 & 363,596 & 14 & 362,120 & $0.00 \%$ & $0.00 \%$ \\
\hline \multirow[t]{5}{*}{$2 \mathrm{~K}$} & 200 & 481 & $1,034,562$ & 53 & $1,033,619$ & 301 & 748,398 & 4 & 735,787 & $27.66 \%$ & $28.81 \%$ \\
\hline & 400 & 177 & 559,957 & 5 & 551,734 & 140 & 533,047 & 6 & 522,706 & $4.81 \%$ & $5.26 \%$ \\
\hline & 600 & 109 & 492,413 & 5 & 489,537 & 103 & 490,971 & 6 & 487,723 & $0.29 \%$ & $0.37 \%$ \\
\hline & 800 & 96 & 485,267 & 6 & 483,083 & 96 & 485,267 & 6 & 483,083 & $0.00 \%$ & $0.00 \%$ \\
\hline & 1000 & 96 & 484,954 & 11 & 483,009 & 96 & 484,954 & 12 & 483,009 & $0.00 \%$ & $0.00 \%$ \\
\hline \multirow[t]{5}{*}{$5 \mathrm{~K}$} & 200 & 481 & $2,477,562$ & 15 & $2,476,619$ & 301 & $1,651,398$ & 4 & $1,638,787$ & $33.35 \%$ & $33.83 \%$ \\
\hline & 400 & 176 & $1,088,244$ & 5 & $1,079,885$ & 127 & 921,786 & 3 & 906,563 & $15.30 \%$ & $16.05 \%$ \\
\hline & 600 & 94 & 786,118 & 18 & 776,513 & 81 & 754,884 & 7 & 746,502 & $3.97 \%$ & $3.86 \%$ \\
\hline & 800 & 67 & 718,496 & 13 & 712,808 & 66 & 717,161 & 12 & 712,080 & $0.19 \%$ & $0.10 \%$ \\
\hline & 1000 & 61 & 707,735 & 18 & 705,568 & 61 & 707,735 & 19 & 705,568 & $0.00 \%$ & $0.00 \%$ \\
\hline \multirow[t]{5}{*}{$10 \mathrm{~K}$} & 200 & 481 & $4,882,562$ & 14 & $4,881,619$ & 301 & $3,156,398$ & 4 & $3,143,787$ & $35.35 \%$ & $35.60 \%$ \\
\hline & 400 & 176 & $1,968,244$ & 6 & $1,959,885$ & 127 & $1,556,786$ & 3 & $1,541,563$ & $20.90 \%$ & $21.34 \%$ \\
\hline & 600 & 92 & $1,248,060$ & 41 & $1,236,696$ & 76 & $1,144,656$ & 7 & $1,134,379$ & $8.29 \%$ & $8.27 \%$ \\
\hline & 800 & 59 & $1,026,556$ & 21 & $1,015,782$ & 56 & $1,015,107$ & 376 & $1,005,689$ & $1.12 \%$ & $0.99 \%$ \\
\hline & 1000 & 48 & 969,852 & 215 & 966,652 & 45 & 964,999 & 208 & 960,718 & $0.50 \%$ & $0.61 \%$ \\
\hline \multirow[t]{5}{*}{$15 \mathrm{~K}$} & 200 & 481 & $7,287,562$ & 16 & $7,286,619$ & 301 & $4,661,398$ & 4 & $4,648,787$ & $36.04 \%$ & $36.20 \%$ \\
\hline & 400 & 176 & $2,848,244$ & 7 & $2,839,885$ & 127 & $2,191,786$ & 4 & $2,176,563$ & $23.05 \%$ & $23.36 \%$ \\
\hline & 600 & 92 & $1,708,060$ & 27 & $1,696,696$ & 73 & $1,513,525$ & 6 & $1,497,946$ & $11.39 \%$ & $11.71 \%$ \\
\hline & 800 & 59 & $1,321,556$ & 2645 & $1,310,782$ & 52 & $1,285,008$ & 47 & $1,275,138$ & $2.77 \%$ & $2.72 \%$ \\
\hline & 1000 & 44 & $1,197,085$ & 9505 & $1,191,986$ & 41 & $1,175,122$ & 370 & $1,170,384$ & $1.83 \%$ & $1.81 \%$ \\
\hline
\end{tabular}

Table 4

Minimum number of facilities.

\begin{tabular}{lllll}
\hline Data & $D_{\max }$ & w/o CIPS & w/ CIPS & \%Diff \\
\hline W287 & 5 & 19 & 14 & $26.32 \%$ \\
& 10 & 7 & 6 & $14.29 \%$ \\
& 15 & 4 & 3 & $25.00 \%$ \\
& 20 & 3 & 2 & $33.33 \%$ \\
& 25 & 2 & 2 & $0.00 \%$ \\
U654 & 200 & 39 & 36 & $7.69 \%$ \\
& 400 & 28 & 17 & $39.29 \%$ \\
& 600 & 15 & 13 & $13.33 \%$ \\
& 800 & 11 & 9 & $18.18 \%$ \\
U1060 & 1000 & 8 & 7 & $12.50 \%$ \\
& 200 & 481 & 301 & $37.42 \%$ \\
& 400 & 176 & 127 & $27.84 \%$ \\
& 600 & 92 & 73 & $20.65 \%$ \\
& 800 & 59 & 50 & $15.25 \%$ \\
& 1000 & 43 & 35 & $18.60 \%$ \\
\hline
\end{tabular}

$600,800,1000\}$ for both U654 and U1060. The $\epsilon$ threshold employed in the stopping criterion of the iterative method in Stage 3 with projections is 0.0001 . Our computational experiments are performed on a dual $2.4 \mathrm{GHz}$ Intel Xeon E5-2630 v3 CPU server with 64GB RAM. The optimization problems that are formed in Matlab R2016a are solved using CPLEX 12.7 in parallel mode using up to 32 threads. We enforce a CPU time limit of ten hours on all our optimization models.

\subsection{Solving the PSCP}

In this section, we present some implementation details about the first stage of our algorithm on the three data sets. The first set of columns in Table 1 present the details of each instance. In this set, we also report the cardinality $C$ of the corresponding CIPS for each instance. The second set of columns present the results obtained by solving the SCP, namely the minimum number of facilities needed to cover all demand points when their locations are selected from the CIPS and the solution CPU times in seconds. Note that some of the demand points may also be included in the CIPS; therefore, the minimum number presents an upper bound on the number of additional points supplied by the PSCP to the second stage. Since the exact solution times are each less than three minutes, we do not propose to implement a heuristic method for the PSCP.

\subsection{Effect of augmenting with the PSCP locations}

In this section, we demonstrate the benefit of adding the PSCP solutions to the set of demand points while forming the set of candidate locations for the discrete problem DLim-PLP.

In Table 2, we present our results for the instances of the data sets W287 and U654. This table is organized as follows: The first set of columns presents the details of each instance. The second set of columns presents the number of facilities $V$, the corresponding costs in the DLim-PLP, and the CPU times in seconds for solving the discrete problem defined over the set of demand points. We also report the cost of the continuous solution obtained at the end of the third stage. The third stage takes less than a second; therefore, we do not report its solution times. The third set of columns presents the same set of results for the discrete problem defined over the set consisting of the demand and the PSCP locations, and its corresponding final solution. In the last set of columns, we show the cost improvements due to the additional candidate locations for both the second and third stage solutions. These improvements are calculated as the difference between the two methods' costs divided by the cost of the former method that does not employ the additional locations from the PSCP solution.

Table 2 indicates that including the PSCP locations in the candidate locations set may lower the number of facilities in the solutions of the DLim-PLP. The decrease in the number of facilities cause substantial improvements in the cost, especially for large $F$ values. Note that augmenting the problem with the additional candidate locations that are obtained from the PSCP resulted with up to $23 \%$ improvements in both the second and the third stage costs for the instances of W287 and U654.

Since the number of additional candidate locations is small compared to the number of demand points, we did not observe a major change in the computation time for the DLim-PLP. The CPU 
Table 5

Third-stage costs and solution times of U1060 instances.

\begin{tabular}{|c|c|c|c|c|c|c|c|c|c|c|c|c|}
\hline \multirow[b]{2}{*}{$\mathrm{F}$} & \multirow[b]{2}{*}{ Dmax } & \multirow{2}{*}{$\frac{\text { Baseline }}{\text { Cost }}$} & \multicolumn{4}{|l|}{ PSCP } & \multicolumn{3}{|c|}{ 40x100 Grid Nodes } & \multicolumn{3}{|c|}{4000 Random CIPS Elements } \\
\hline & & & Added & Cost & $\%$ Diff & Time & Cost & \% Diff & Time & Min Cost & \% Diff & Time \\
\hline 1000 & 200 & 552,619 & 301 & 434,834 & $21.31 \%$ & 14 & 488,931 & $11.52 \%$ & 1029 & 469,237 & $15.09 \%$ & 32,486 \\
\hline 1000 & 400 & 373,288 & 127 & 370,294 & $0.80 \%$ & 18 & 367,943 & $1.43 \%$ & 52 & 372,400 & $0.24 \%$ & 879 \\
\hline 1000 & 600 & 363,314 & 73 & 363,270 & $0.01 \%$ & 33 & 362,901 & $0.11 \%$ & 138 & 363,157 & $0.04 \%$ & 1037 \\
\hline 1000 & 800 & 362,127 & 50 & 362,127 & $0.00 \%$ & 186 & 361,978 & $0.04 \%$ & 158 & 362,008 & $0.03 \%$ & 1156 \\
\hline 1000 & 1000 & 362,120 & 35 & 362,120 & $0.00 \%$ & 116 & 361,971 & $0.04 \%$ & 146 & 361,958 & $0.04 \%$ & 8476 \\
\hline 2000 & 200 & $1,033,619$ & 301 & 735,787 & $28.81 \%$ & 4 & 881,931 & $14.68 \%$ & 1398 & 825,649 & $20.12 \%$ & 39,434 \\
\hline 2000 & 400 & 551,734 & 127 & 522,706 & $5.26 \%$ & 19 & 526,165 & $4.63 \%$ & 31 & 550,138 & $0.29 \%$ & 1041 \\
\hline 2000 & 600 & 489,537 & 73 & 487,723 & $0.37 \%$ & 34 & 486,904 & $0.54 \%$ & 169 & 489,043 & $0.10 \%$ & 1278 \\
\hline 2000 & 800 & 483,083 & 50 & 483,083 & $0.00 \%$ & 187 & 482,963 & $0.02 \%$ & 192 & 483,082 & $0.00 \%$ & 1707 \\
\hline 2000 & 1000 & 483,009 & 35 & 483,009 & $0.00 \%$ & 114 & 482,960 & $0.01 \%$ & 189 & 482,997 & $0.00 \%$ & 8472 \\
\hline 5000 & 200 & $2,476,619$ & 301 & $1,638,787$ & $33.83 \%$ & 4 & $2,060,931$ & $16.78 \%$ & 1007 & $1,893,649$ & $23.54 \%$ & 48,199 \\
\hline 5000 & 400 & $1,079,885$ & 127 & 906,563 & $16.05 \%$ & 16 & 970,005 & $10.18 \%$ & 1120 & $1,055,923$ & $2.22 \%$ & 6849 \\
\hline 5000 & 600 & 776,513 & 73 & 746,502 & $3.86 \%$ & 35 & 756,631 & $2.56 \%$ & 1730 & 770,880 & $0.73 \%$ & 29,508 \\
\hline 5000 & 800 & 712,808 & 50 & 712,080 & $0.10 \%$ & 193 & 709,349 & $0.49 \%$ & 182 & 712,803 & $0.00 \%$ & 4256 \\
\hline 5000 & 1000 & 705,568 & 35 & 705,568 & $0.00 \%$ & 121 & 704,611 & $0.14 \%$ & 237 & 705,417 & $0.02 \%$ & 11,297 \\
\hline 10000 & 200 & $4,881,619$ & 301 & $3,143,787$ & $35.60 \%$ & 4 & $4,025,931$ & $17.53 \%$ & 1080 & $3,673,649$ & $24.75 \%$ & 45,252 \\
\hline 10000 & 400 & $1,959,885$ & 127 & $1,541,563$ & $21.34 \%$ & 16 & $1,705,005$ & $13.00 \%$ & 15,465 & $1,900,923$ & $3.01 \%$ & 6097 \\
\hline 10000 & 600 & $1,236,696$ & 73 & $1,134,379$ & $8.27 \%$ & 35 & $1,175,393$ & $4.96 \%$ & 36,000 & $1,221,063$ & $1.26 \%$ & 33,724 \\
\hline 10000 & 800 & $1,015,782$ & 50 & $1,005,689$ & $0.99 \%$ & 557 & 997,056 & $1.84 \%$ & 492 & $1,011,628$ & $0.41 \%$ & 8873 \\
\hline 10000 & 1000 & 966,652 & 35 & 960,718 & $0.61 \%$ & 310 & 958,732 & $0.82 \%$ & 710 & 966,301 & $0.04 \%$ & 93,303 \\
\hline 15000 & 200 & $7,286,619$ & 301 & $4,648,787$ & $36.20 \%$ & 4 & $5,990,931$ & $17.78 \%$ & 1174 & $5,453,649$ & $25.16 \%$ & 31,398 \\
\hline 15000 & 400 & $2,839,885$ & 127 & $2,176,563$ & $23.36 \%$ & 17 & $2,440,005$ & $14.08 \%$ & 36,000 & $2,745,923$ & $3.31 \%$ & 3996 \\
\hline 15000 & 600 & $1,696,696$ & 73 & $1,497,946$ & $11.71 \%$ & 34 & $1,595,861$ & $5.94 \%$ & 36,000 & $1,671,063$ & $1.51 \%$ & 38,096 \\
\hline 15000 & 800 & $1,310,782$ & 50 & $1,275,138$ & $2.72 \%$ & 228 & $1,265,060$ & $3.49 \%$ & 26,673 & $1,301,628$ & $0.70 \%$ & 488,856 \\
\hline 15000 & 1000 & $1,191,986$ & 35 & $1,170,384$ & $1.81 \%$ & 472 & $1,166,401$ & $2.15 \%$ & 22,454 & $1,187,091$ & $0.41 \%$ & $1,317,529$ \\
\hline
\end{tabular}

times of both models were comparable. We report the solutions for the data set U1060 in Table 3, which is organized in the same way as Table 2.

Table 3 also indicates that the solution times of both models are comparable. As also observed in the instances of W287 and U654, assuming the PSCP solutions as possible locations for the facilities decreased the number of facilities needed considerably. In the instances of U1060 that we present, we observe cost differences of up to $36 \%$ in both the second and the third stages.

To explain such big differences in the cost, we present in Table 4 the minimum number of facilities needed to cover all demand points under both candidate location sets. As the $F$ value is increased, the cost difference percentages approach to the difference percentages presented in this table. Hence, we view the decrease in the number of facilities as the main reason for the cost improvements.

\subsection{Effect of augmenting with arbitrary locations}

Additional candidate locations in the DLim-PLP is expected to lower the cost as we work with a larger feasible set. In the following analysis, we show that the number of additional locations obtained from the PSCP solution is small; however, the cost improvement is substantial compared to the size of the additional locations set.

In Table 5, we compare three different sets of additional candidate locations in terms of the third-stage cost improvements and the solution times on the instances of U1060. Our baseline has no additional candidate locations. The first set is formed of the locations in the PSCP solution. For the second set, we overlay a grid of $40 \times 100$ on the area containing the demand points and form the set composed of the 4000 grid nodes. The last set is composed of 4000 random elements from the set of circle intersection points. Since the result would depend on the selected random locations, we form 100 such random sets and report the best cost obtained for each instance. Since 4000 additional candidate locations increase the size of the problem considerably, we implement a CPU time limit of $10 \mathrm{~h}$ for each CPLEX solution.
Table 5 is organized as follows: The first set of columns presents the parameters of the instances. The third column presents the baseline cost, which is determined by solving the DLim-PLP with the demand locations as the only candidate locations for the facilities and then by fine-tuning the facility locations using our method with projections that we employ in the third stage of our heuristic method. The third set of columns present the number of additional candidate locations, the resulting costs, the percentage improvements, and the total solution times of our method. The number of additional candidate locations for the other two methods are 4000 for each instance, hence we do not include this information in the table. In the fourth and fifth sets of columns, we present the costs, the percentage improvements and the solution times for the methods adding the random locations and the grid nodes, respectively. Note that the fine-tuning method is also applied to the methods with the random locations and the grid nodes. The percentage improvements are calculated as the decrease in the cost divided by the cost in the third column. For each instance, we indicate the best method by a boldface entry.

Table 5 indicates that, even though the number of additional candidate locations is a lot smaller, our proposed method outperforms the other two alternatives when $D_{\max }$ is small and $F$ is large. In these instances, the facility costs are dominant and our method picks the locations to minimize the number of facilities, while the other two alternatives cannot. Moreover, our method's solution times are substantially smaller for these instances. In the instances where the other methods outperform our method, their costs are at most $1 \%$ lower.

\subsection{Performance of our iterative heuristic method with projections}

In the third stage of our heuristic solution method, we apply Cooper's alternating location and allocation algorithm. In the location step of this algorithm, instead of solving the DLim-Geom, we employ an iterative heuristic method with projections. To demonstrate the performance of this method, we also obtained results for the instances reported in Tables 2 and 3 by solving the DLim- 
Table 6

Heuristic performance on W287 and U654.

\begin{tabular}{|c|c|c|c|c|c|c|c|c|c|c|c|c|}
\hline \multirow[b]{3}{*}{ Data } & \multirow[b]{3}{*}{$\mathrm{F}$} & \multirow[b]{3}{*}{$D_{\max }$} & \multicolumn{5}{|c|}{ w/o PSCP } & \multicolumn{5}{|l|}{$\mathrm{w} / \mathrm{PSCP}$} \\
\hline & & & \multicolumn{2}{|c|}{ DLim-Geom } & \multicolumn{2}{|l|}{ Heuristic } & \multirow{2}{*}{$\begin{array}{l}\text { Cost } \\
\text { Diff }\end{array}$} & \multicolumn{2}{|c|}{ DLim-Geom } & \multicolumn{2}{|l|}{ Heuristic } & \multirow{2}{*}{$\begin{array}{l}\text { Cost } \\
\text { Diff }\end{array}$} \\
\hline & & & Cost & Time & Cost & Time & & Cost & Time & Cost & Time & \\
\hline \multirow[t]{5}{*}{ W287 } & 50 & 5 & 4144 & 32.93 & 4157 & 0.24 & $0.30 \%$ & 4022 & 24.16 & 4033 & 0.13 & $0.27 \%$ \\
\hline & 50 & 10 & 3946 & 22.91 & 3951 & 0.03 & $0.13 \%$ & 3921 & 18.94 & 3926 & 0.03 & $0.14 \%$ \\
\hline & 50 & 15 & 3885 & 20.90 & 3889 & 0.03 & $0.11 \%$ & 3885 & 19.27 & 3889 & 0.02 & $0.11 \%$ \\
\hline & 50 & 20 & 3885 & 18.73 & 3889 & 0.03 & $0.11 \%$ & 3885 & 16.45 & 3889 & 0.02 & $0.11 \%$ \\
\hline & 50 & 25 & 3880 & 18.68 & 3884 & 0.04 & $0.11 \%$ & 3880 & 19.60 & 3884 & 0.02 & $0.11 \%$ \\
\hline \multirow[t]{5}{*}{ W287 } & 100 & 5 & 6492 & 24.36 & 6499 & 0.03 & $0.11 \%$ & 6255 & 19.06 & 6272 & 0.02 & $0.27 \%$ \\
\hline & 100 & 10 & 5925 & 20.65 & 5932 & 0.01 & $0.10 \%$ & 5850 & 21.60 & 5857 & 0.03 & $0.11 \%$ \\
\hline & 100 & 15 & 5764 & 16.45 & 5770 & 0.03 & $0.11 \%$ & 5764 & 16.52 & 5770 & 0.01 & $0.11 \%$ \\
\hline & 100 & 20 & 5764 & 21.48 & 5770 & 0.02 & $0.11 \%$ & 5764 & 20.92 & 5770 & 0.03 & $0.11 \%$ \\
\hline & 100 & 25 & 5710 & 21.40 & 5717 & 0.02 & $0.11 \%$ & 5710 & 19.86 & 5717 & 0.02 & $0.11 \%$ \\
\hline \multirow[t]{5}{*}{ W287 } & 200 & 5 & 10,197 & 23.91 & 10,220 & 0.02 & $0.23 \%$ & 9608 & 19.09 & 9632 & 0.02 & $0.25 \%$ \\
\hline & 200 & 10 & 8724 & 12.75 & 8741 & 0.02 & $0.19 \%$ & 8608 & 8.17 & 8619 & 0.02 & $0.14 \%$ \\
\hline & 200 & 15 & 8348 & 11.36 & 8357 & 0.02 & $0.11 \%$ & 8348 & 10.20 & 8357 & 0.02 & $0.11 \%$ \\
\hline & 200 & 20 & 8296 & 13.35 & 8305 & 0.03 & $0.10 \%$ & 8296 & 11.32 & 8305 & 0.02 & $0.10 \%$ \\
\hline & 200 & 25 & 8147 & 10.85 & 8157 & 0.02 & $0.12 \%$ & 8147 & 9.86 & 8157 & 0.02 & $0.12 \%$ \\
\hline \multirow[t]{5}{*}{ W287 } & 500 & 5 & 17,876 & 18.28 & 17,905 & 0.03 & $0.16 \%$ & 15,986 & 15.21 & 16,004 & 0.01 & $0.11 \%$ \\
\hline & 500 & 10 & 13,587 & 10.67 & 13,609 & 0.01 & $0.16 \%$ & 13,206 & 9.55 & 13,228 & 0.01 & $0.17 \%$ \\
\hline & 500 & 15 & 12,283 & 7.41 & 12,290 & 0.03 & $0.05 \%$ & 12,283 & 7.11 & 12,290 & 0.02 & $0.05 \%$ \\
\hline & 500 & 20 & 12,107 & 8.64 & 12,113 & 0.01 & $0.05 \%$ & 12,001 & 8.32 & 12,024 & 0.01 & $0.19 \%$ \\
\hline & 500 & 25 & 11,617 & 6.20 & 11,635 & 0.02 & $0.15 \%$ & 11,617 & 5.59 & 11,635 & 0.01 & $0.15 \%$ \\
\hline \multirow{5}{*}{ W287 } & 5000 & 5 & 105,933 & 18.40 & 106,008 & 0.02 & $0.07 \%$ & 82,867 & 9.85 & 82,867 & 0.02 & $0.00 \%$ \\
\hline & 5000 & 10 & 52,851 & 10.35 & 52,903 & 0.02 & $0.10 \%$ & 48,384 & 7.54 & 48,453 & 0.01 & $0.14 \%$ \\
\hline & 5000 & 15 & 35,581 & 13.77 & 35,670 & 0.02 & $0.25 \%$ & 35,555 & 7.07 & 35,603 & 0.02 & $0.14 \%$ \\
\hline & 5000 & 20 & 31,203 & 11.19 & 31,217 & 0.02 & $0.05 \%$ & 27,484 & 32.41 & 27,555 & 0.04 & $0.26 \%$ \\
\hline & 5000 & 25 & 26,638 & 13.91 & 26,837 & 0.01 & $0.75 \%$ & 26,638 & 14.17 & 26,837 & 0.01 & $0.75 \%$ \\
\hline U654 & 1000 & 200 & 80,547 & 29.72 & 80,545 & 0.17 & $0.00 \%$ & 78,576 & 22.78 & 78,661 & 0.24 & $0.11 \%$ \\
\hline & 1000 & 400 & 77,099 & 27.60 & 77,099 & 0.04 & $0.00 \%$ & 75,289 & 20.44 & 75,292 & 0.06 & $0.00 \%$ \\
\hline & 1000 & 600 & 74,680 & 26.08 & 74,687 & 0.05 & $0.01 \%$ & 74,678 & 20.06 & 74,686 & 0.04 & $0.01 \%$ \\
\hline & 1000 & 800 & 73,968 & 26.73 & 73,968 & 0.06 & $0.00 \%$ & 73,968 & 20.18 & 73,968 & 0.06 & $0.00 \%$ \\
\hline & 1000 & 1000 & 73,968 & 24.65 & 73,968 & 0.05 & $0.00 \%$ & 73,968 & 21.05 & 73,968 & 0.04 & $0.00 \%$ \\
\hline U654 & 2000 & 200 & 125,298 & 17.11 & 125,297 & 0.03 & $0.00 \%$ & 120,328 & 15.79 & 120,412 & 0.04 & $0.07 \%$ \\
\hline & 2000 & 400 & 115,451 & 15.62 & 115,451 & 0.02 & $0.00 \%$ & 108,164 & 14.59 & 108,167 & 0.05 & $0.00 \%$ \\
\hline & 2000 & 600 & 103,787 & 15.13 & 103,794 & 0.04 & $0.01 \%$ & 103,787 & 14.49 & 103,794 & 0.04 & $0.01 \%$ \\
\hline & 2000 & 800 & 102,613 & 14.64 & 102,660 & 0.04 & $0.05 \%$ & 102,613 & 14.57 & 102,660 & 0.05 & $0.05 \%$ \\
\hline & 2000 & 1000 & 102,219 & 14.19 & 102,246 & 0.04 & $0.03 \%$ & 102,217 & 14.36 & 102,246 & 0.04 & $0.03 \%$ \\
\hline U654 & 5000 & 200 & 251,682 & 17.71 & 251,681 & 0.04 & $0.00 \%$ & 237,712 & 13.75 & 237,796 & 0.01 & $0.04 \%$ \\
\hline & 5000 & 400 & 212,154 & 11.51 & 212,154 & 0.01 & $0.00 \%$ & 184,566 & 11.63 & 184,653 & 0.03 & $0.05 \%$ \\
\hline & 5000 & 600 & 170,321 & 13.23 & 170,441 & 0.02 & $0.07 \%$ & 169,115 & 13.47 & 169,336 & 0.03 & $0.13 \%$ \\
\hline & 5000 & 800 & 163,070 & 12.12 & 163,174 & 0.02 & $0.06 \%$ & 161,339 & 13.56 & 161,454 & 0.02 & $0.07 \%$ \\
\hline & 5000 & 1000 & 155,345 & 11.31 & 155,456 & 0.04 & $0.07 \%$ & 155,343 & 16.44 & 155,456 & 0.03 & $0.07 \%$ \\
\hline U654 & 10000 & 200 & 446,682 & 21.97 & 446,681 & 0.03 & $0.00 \%$ & 417,712 & 14.05 & 417,796 & 0.01 & $0.02 \%$ \\
\hline & 10000 & 400 & 352,154 & 13.76 & 352,154 & 0.02 & $0.00 \%$ & 283,890 & 14.17 & 284,128 & 0.04 & $0.08 \%$ \\
\hline & 10000 & 600 & 255,562 & 16.25 & 255,742 & 0.02 & $0.07 \%$ & 248,812 & 13.35 & 249,352 & 0.03 & $0.22 \%$ \\
\hline & 10000 & 800 & 238,070 & 11.74 & 238,174 & 0.02 & $0.04 \%$ & 228,612 & 15.78 & 229,700 & 0.04 & $0.48 \%$ \\
\hline & 10000 & 1000 & 219,636 & 11.13 & 219,752 & 0.03 & $0.05 \%$ & 219,636 & 9.05 & 219,752 & 0.03 & $0.05 \%$ \\
\hline U654 & 15000 & 200 & 641,682 & 21.06 & 641,681 & 0.04 & $0.00 \%$ & 597,712 & 13.61 & 597,796 & 0.02 & $0.01 \%$ \\
\hline & 15000 & 400 & 492,154 & 13.22 & 492,154 & 0.03 & $0.00 \%$ & 378,473 & 15.86 & 378,753 & 0.03 & $0.07 \%$ \\
\hline & 15000 & 600 & 335,754 & 16.10 & 336,046 & 0.02 & $0.09 \%$ & 324,004 & 14.04 & 324,656 & 0.04 & $0.20 \%$ \\
\hline & 15000 & 800 & 313,070 & 11.86 & 313,174 & 0.02 & $0.03 \%$ & 294,209 & 9.44 & 295,492 & 0.01 & $0.44 \%$ \\
\hline & 15000 & 1000 & 279,525 & 11.07 & 279,641 & 0.02 & $0.04 \%$ & 279,525 & 10.57 & 279,641 & 0.04 & $0.04 \%$ \\
\hline
\end{tabular}

Geom at the location steps of Cooper's algorithm. The $\epsilon$ threshold is again taken as 0.0001 .

Tables 6 and 7 present the results for the data sets W287, U654, and U1060. In these tables, the first set of columns present the details of the instance. The next set of columns present the solutions from both solving the DLim-Geom and applying the heuristic in the location steps along with the solution times in CPU seconds for the method not employing the PSCP solutions. The last column of this set presents the increase in the cost due to employing the heuristic instead of solving DLim-Geom. The last set of columns present the same information for the our solution method employing the PSCP solutions.

Tables 6 and 7 demonstrate that employing our iterative method with projections instead of solving the DLim-Geom increases the final cost by at most $0.75 \%$. Moreover, the heuristic method obtains these solutions hundreds of times faster than solv- ing the DLim-Geom. Hence, we propose this heuristic method as a decent alternative to solving the DLim-Geom. Note that when the DLim-Geom is solved at the location steps, the longest solution time of the third stage over all the instances of these three data sets is a little over four minutes, which may also be acceptable for planning purposes.

\section{Conclusion}

We introduced a new continuous location-allocation problem with a distance limitation that is applicable to water and energy distribution systems. We presented a MIQCP formulation and proposed a heuristic solution method. Our heuristic method is based on solving a discrete version of the problem to obtain an initial solution for the Cooper's algorithm that obtains a local optimum solution in the continuous space. The candidate facility locations of discrete version of the problem included not only the demand 
Table 7

Heuristic performance on U1060.

\begin{tabular}{|c|c|c|c|c|c|c|c|c|c|c|c|}
\hline \multirow[b]{3}{*}{$\mathrm{F}$} & \multirow[b]{3}{*}{$D_{\max }$} & \multicolumn{5}{|l|}{ w/o PSCP } & \multicolumn{5}{|l|}{ w/ PSCP } \\
\hline & & \multicolumn{2}{|c|}{ DLim-Geom } & \multicolumn{2}{|l|}{ Heuristic } & \multirow{2}{*}{$\begin{array}{l}\text { Cost } \\
\text { Diff }\end{array}$} & \multicolumn{2}{|c|}{ DLim-Geom } & \multicolumn{2}{|l|}{ Heuristic } & \multirow{2}{*}{$\begin{array}{l}\text { Cost } \\
\text { Diff }\end{array}$} \\
\hline & & Cost & Time & Cost & Time & & Cost & Time & Cost & Time & \\
\hline 1000 & 200 & 552,579 & 188.87 & 552,619 & 0.38 & $0.01 \%$ & 434,618 & 184.04 & 434,834 & 0.33 & $0.05 \%$ \\
\hline 1000 & 400 & 373,234 & 164.23 & 373,288 & 0.21 & $0.01 \%$ & 370,021 & 80.52 & 370,294 & 0.16 & $0.07 \%$ \\
\hline 1000 & 600 & 363,308 & 77.29 & 363,314 & 0.11 & $0.00 \%$ & 363,261 & 71.97 & 363,270 & 0.14 & $0.00 \%$ \\
\hline 1000 & 800 & 362,125 & 85.08 & 362,127 & 0.14 & $0.00 \%$ & 362,125 & 71.27 & 362,127 & 0.12 & $0.00 \%$ \\
\hline 1000 & 1000 & 362,118 & 84.49 & 362,120 & 0.14 & $0.00 \%$ & 362,118 & 71.43 & 362,120 & 0.11 & $0.00 \%$ \\
\hline 2000 & 200 & $1,033,579$ & 215.68 & $1,033,619$ & 0.25 & $0.00 \%$ & 735,546 & 188.54 & 735,787 & 0.15 & $0.03 \%$ \\
\hline 2000 & 400 & 551,577 & 145.70 & 551,734 & 0.20 & $0.03 \%$ & 521,935 & 84.45 & 522,706 & 0.11 & $0.15 \%$ \\
\hline 2000 & 600 & 489,413 & 60.06 & 489,537 & 0.06 & $0.03 \%$ & 487,278 & 63.69 & 487,723 & 0.06 & $0.09 \%$ \\
\hline 2000 & 800 & 483,082 & 56.15 & 483,083 & 0.07 & $0.00 \%$ & 483,082 & 59.33 & 483,083 & 0.06 & $0.00 \%$ \\
\hline 2000 & 1000 & 483,009 & 59.86 & 483,009 & 0.07 & $0.00 \%$ & 483,009 & 60.01 & 483,009 & 0.09 & $0.00 \%$ \\
\hline 5000 & 200 & $2,476,579$ & 243.58 & $2,476,619$ & 0.18 & $0.00 \%$ & $1,638,546$ & 223.74 & $1,638,787$ & 0.13 & $0.01 \%$ \\
\hline 5000 & 400 & $1,079,720$ & 175.33 & $1,079,885$ & 0.17 & $0.02 \%$ & 905,200 & 82.16 & 906,563 & 0.07 & $0.15 \%$ \\
\hline 5000 & 600 & 776,492 & 87.40 & 776,513 & 0.09 & $0.00 \%$ & 745,148 & 49.89 & 746,502 & 0.06 & $0.18 \%$ \\
\hline 5000 & 800 & 712,719 & 65.93 & 712,808 & 0.07 & $0.01 \%$ & 712,002 & 48.35 & 712,080 & 0.07 & $0.01 \%$ \\
\hline 5000 & 1000 & 705,501 & 49.94 & 705,568 & 0.07 & $0.01 \%$ & 705,501 & 37.62 & 705,568 & 0.07 & $0.01 \%$ \\
\hline 10000 & 200 & $4,881,579$ & 244.54 & $4,881,619$ & 0.25 & $0.00 \%$ & $3,143,546$ & 172.2 & $3,143,787$ & 0.13 & $0.01 \%$ \\
\hline 10000 & 400 & $1,959,720$ & 174.74 & $1,959,885$ & 0.20 & $0.01 \%$ & $1,540,200$ & 75.44 & $1,541,563$ & 0.06 & $0.09 \%$ \\
\hline 10000 & 600 & $1,235,705$ & 85.01 & $1,236,696$ & 0.07 & $0.08 \%$ & $1,132,724$ & 46.98 & $1,134,379$ & 0.08 & $0.15 \%$ \\
\hline 10000 & 800 & $1,014,142$ & 106.08 & $1,015,782$ & 0.06 & $0.16 \%$ & $1,005,410$ & 66.6 & $1,005,689$ & 0.1 & $0.03 \%$ \\
\hline 10000 & 1000 & 966,356 & 33.20 & 966,652 & 0.04 & $0.03 \%$ & 960,403 & 28.31 & 960,718 & 0.05 & $0.03 \%$ \\
\hline 15000 & 200 & $7,286,579$ & 243.34 & $7,286,619$ & 0.24 & $0.00 \%$ & $4,648,546$ & 160.81 & $4,648,787$ & 0.14 & $0.01 \%$ \\
\hline 15000 & 400 & $2,839,720$ & 176.44 & $2,839,885$ & 0.19 & $0.01 \%$ & $2,175,200$ & 97.86 & $2,176,563$ & 0.11 & $0.06 \%$ \\
\hline 15000 & 600 & $1,695,705$ & 85.16 & $1,696,696$ & 0.10 & $0.06 \%$ & $1,496,223$ & 67.95 & $1,497,946$ & 0.07 & $0.12 \%$ \\
\hline 15000 & 800 & $1,309,142$ & 106.14 & $1,310,782$ & 0.05 & $0.13 \%$ & $1,273,352$ & 49.23 & $1,275,138$ & 0.08 & $0.14 \%$ \\
\hline 15000 & 1000 & $1,191,263$ & 51.17 & $1,191,986$ & 0.06 & $0.06 \%$ & $1,169,890$ & 43.09 & $1,170,384$ & 0.04 & $0.04 \%$ \\
\hline
\end{tabular}

locations but also the locations in the PSCP solution under the distance limitation. Even though the number of additional candidate locations is small, we observed substantial drops in the costs of the instances with tight distance constraints, as it was feasible to serve all demand points with fewer facilities. As the coverage distance gets larger, the number of additional candidate locations gets smaller; therefore, the benefit of the method diminishes.

The location step of Cooper's algorithm, which utilized Weiszfeld's method, was also modified to incorporate the distance limitation. We proposed a projection method to preserve feasibility at every iteration.

The first two stages of our three-stage heuristic method yield clusters of demand points and a facility location for each cluster. Rather than solving two IP problems, these two stages can be replaced by a clustering method. Alternative heuristic methods based on clustering are currently under investigation. Another research direction is to consider facilities with limited capacities. The additional capacity constraint may be handled by modifying the IP problem in the second stage, and modifying the allocation step of Cooper's algorithm. A final possible research direction is to change the type of the facilities from decentralized to centralized. In that case, a two-level network design problem will be considered and the heuristic solutions can be built upon the foundation presented in this paper.

\section{Acknowledgements}

We gratefully acknowledge the insightful comments and suggestions of two anonymous reviewers and the editors, which led to several improvements in our manuscript.

\section{References}

Balas, E., Carrera, M.C., 1996. A dynamic subgradient-based branch-and-bound procedure for set covering. Oper. Res. 44 (6), 875-890.

Bautista, J., Pereira, J., 2007. A grasp algorithm to solve the unicost set covering problem. Comput. Oper. Res. 34 (10), 3162-3173.

Beasley, J.E., 1987. An algorithm for set covering problem. Eur. J. Oper. Res. 31 (1), 85-93.
Beasley, J.E., 1990. A lagrangian heuristic for set-covering problems. Nav. Res. Logist. (NRL) 37 (1), 151-164.

Beasley, J.E., Chu, P.C., 1996. A genetic algorithm for the set covering problem. Eur. J. Oper. Res. 94 (2), 392-404.

Beasley, J.E., Jörnsten, K., 1992. Enhancing an algorithm for set covering problems. Eur. J. Oper. Res. 58 (2), 293-300.

Berman, O., Yang, E.K., 1991. Medi-centre location problems. J. Oper. Res. Soc. 42 (4) 313-322.

Bongartz, I., Calamai, P.H., Conn, A.R., 1994. A projection method for Lp norm location-allocation problems. Math. Program. 66 (1-3), 283-312.

Brimberg, J., Drezner, Z., 2013. A new heuristic for solving the p-median problem in the plane. Comput. Oper. Res. 40 (1), 427-437.

Brimberg, J., Drezner, Z., Mladenović, N., Salhi, S., 2014. A new local search for continuous location problems. Eur. J. Oper. Res. 232 (2), 256-265.

Brimberg, J., Hansen, P., Mladenovic, N., Salhi, S., 2008. A survey of solution methods for the continuous location-allocation problem. Int. J. Oper. Res. 5 (1), 1-12.

Brimberg, J., Mladenovic, N., Salhi, S., 2004. The multi-source Weber problem with constant opening cost. J. Oper. Res. Soc. 55, 640-646.

Brimberg, J., Salhi, S., 2005. A continuous location-allocation problem with zonedependent fixed cost. Ann. Oper. Res. 136 (1), 99-115.

Caprara, A., Fischetti, M., Toth, P., 1999. A heuristic method for the set covering problem. Oper. Res. 47 (5), 730-743.

Caprara, A., Toth, P., Fischetti, M., 2000. Algorithms for the set covering problem. Ann. Oper. Res. 98 (1-4), 353-371.

Church, R.L., 1984. The planar maximal covering location problem. J. Reg. Sci. 24 (2), 185-201.

Cooper, L., 1963. Location-allocation problems. Oper. Res. 11 (3), 331-343.

Cooper, L., 1964. Heuristic methods for location-allocation problems. SIAM Rev. 6 (1), 37-53.

Douglas, J., Gasiorek, J., Swaffield, J., 1979. Fluid Mechanics. 1995. Longman Group Ltd.

Drezner, Z., Brimberg, J., Mladenović, N., Salhi, S., 2015. New heuristic algorithms for solving the planar p-median problem. Comput. Oper. Res. 62, 296-304.

Drezner, Z., Brimberg, J., Mladenović, N., Salhi, S., 2016. New local searches for solving the multi-source Weber problem. Ann. Oper. Res. 246 (1-2), 181-203.

Drezner, Z., Mehrez, A., Wesolowsky, G.O., 1991. The facility location problem with limited distances. Transp. Sci. 25 (3), 183-187.

Eiselt, H.A., Sandblom, C.-L., 2013. Decision Analysis, Location Models, and Scheduling Problems. Springer Science \& Business Media.

Fernandes, I.F., Aloise, D., Aloise, D.J., Hansen, P., Liberti, L., 2014. On the weber facility location problem with limited distances and side constraints. Optim. Lett. 8, 407-424

Fisher, M.L., Kedia, P., 1990. Optimal solution of set covering/partitioning problems using dual heuristics. Manage. Sci. 36 (6), 674-688.

Garey, M.R., Johnson, D.S., 1979. Computers and Intractability AGuide to the Theory of NP-Completeness, 58. Freeman, San Francisco, LA, p. 1979.

Haddadi, S., 1997. Simple Lagrangian heuristic for the set covering problem. Eur. J. Oper. Res. 97 (1), 200-204. 
Hansen, P., Mladenović, N., Taillard, E., 1998. Heuristic solution of the multisource Weber problem as a p-median problem. Oper. Res. Lett. 22 (2), 55-62.

Haouari, M., Chaouachi, J., 2002. A probabilistic greedy search algorithm for combinatorial optimisation with application to the set covering problem. J. Oper. Res. Soc. 53 (7), 792-799.

Kariv, O., Hakimi, S.L., 1979. An algorithmic approach to network location problems. ii: the p-medians. SIAM J. Appl. Math. 37 (3), 539-560.

Kocaman, A.S., Huh, W.T., Modi, V., 2012. Initial layout of power distribution systems for rural electrification: a heuristic algorithm for multilevel network design. Appl. Energy 96, 302-315.

Krarup, J., Pruzan, P.M., 1983. The simple plant location problem: survey and synthesis. Eur. J. Oper. Res. 12 (1), 36-81.

Krysta, P., Solis-Oba., R., 2001. Approximation algorithms for bounded facility location problems. J. Comb. Optim. 5, 233-247.

Lan, G., DePuy, G.W., Whitehouse, G.E., 2007. An effective and simple heuristic for the set covering problem. Eur. J. Oper. Res. 176 (3), 1387-1403.

Lorena, L.A.N., Lopes, F.B., 1994. A surrogate heuristic for set covering problems. Eur. J. Oper. Res. 79 (1), 138-150.

Love, R., Morris, J., Wesolowsky, G., 1988. Facilities Location: Models and Methods. North-Holland, New York.

Megiddo, N., Supowit, K.J., 1984. On the complexity of some common geometric location problems. SIAM J. Comput. 13 (1), 182-196.
Rajagopalan, H.K., Saydam, C., Xiao, J., 2008. A multiperiod set covering location model for dynamic redeployment of ambulances. Comput. Oper. Res. 35 (3), 814-826.

Reinelt, G., 1991. TSPLIB-a traveling salesman problem library. ORSA J. Comput. 3 (4), 376-384.

Ren, Z.-G., Feng, Z.-R., Ke, L.-J., Zhang, Z.-J., 2010. New ideas for applying ant colony optimization to the set covering problem. Comput. Ind. Eng. 58 (4), 774-784.

Solar, M., Parada, V., Urrutia, R., 2002. A parallel genetic algorithm to solve the setcovering problem. Comput. Oper. Res. 29 (9), 1221-1235.

Toregas, C., Swain, R., ReVelle, C., Bergman, L., 1971. The location of emergency service facilities. Oper. Res. 19 (6), 1363-1373.

Vardi, Y., Zhang, C.-H., 2001. A modified Weiszfeld algorithm for the Fermat-Weber location problem. Math. Program. 90 (3), 559-566.

Weber, A., 1929. Über den Standort der Industrien. Tübingen: JCB Mohr. English Translation: The Theory of the Location of Industries. Chicago University Press, Chicago.

Weiszfeld, E., 1937. Sur le point pour lequel la somme des distances de $\mathrm{n}$ points donnés est minimum. Tohoku Math. J. 43 (2), 355-386.

Weng, K., 2013. Approximation algorithm for uniform bounded facility location problem. J. Comb. Optim. 26, 284-291.

Yagiura, M., Kishida, M., Ibaraki, T., 2006. A 3-flip neighborhood local search for the set covering problem. Eur. J. Oper. Res. 172 (2), 472-499. 